FOLIATIONS 2005

ed. by Paweł WALCZAK et al.

World Scientific, Singapore, 2006

pp. $71-108$

\title{
HIRSCH FOLIATIONS IN CODIMENSION GREATER
} THAN ONE

\author{
ANDRZEJ BIŚ \\ Wydziat Matematyki, Uniwersytet Eódzki \\ Banacha 22, 90-238, Eódź, Poland \\ e-mail: andbis@math.uni.lodz.pl \\ STEVEN HURDER \\ Department of Mathematics ( $m / c$ 249), University of Illinois at Chicago \\ 851 S. Morgan St. CHICAGO, IL 60607-7045, USA \\ e-mail: hurder@uic.edu,Web: http://www.math.uic.edu/ hurder/ \\ JOSEPH SHIVE \\ Department of Mathematics, Richard J. Daley College, \\ 7500 S. Pulaski Road, CHICAGO, IL 60652 USA \\ e-mail: jshive@ccc.edu

\begin{abstract}
We generalize the Hirsch construction of a smooth foliation on a 3-manifold with a unique exceptional minimal set, to obtain a method for constructing smooth foliations of arbitrary codimension with exotic minimal sets. The method also yields a procedure to realize a given system of étale correspondences as the holonomy of a smooth foliation of a compact manifold. This generalizes the well-known group suspension construction.
\end{abstract}

\section{Introduction}

The "Hirsch foliation", as originally constructed by Morris Hirsch in [36], is an analytic codimension one foliation of a compact 3-manifold $N$ with 
a unique minimal set $\mathbf{K}$ of exceptional type. All of the leaves of $\mathcal{F}$ in $\mathbf{K}$ have exponential volume growth rate, and there is a countable set of leaves with non-trivial holonomy, generated by a single contraction. This foliation admits a complete closed transversal diffeomorphic to $\mathbb{S}^{1}$, but the global holonomy of the foliation is not equivalent to a group acting on $\mathbb{S}^{1}$. The procedure for constructing the Hirsch foliation is actually a recipe for constructing many families of foliations, depending on the choices made. For example, the literature often considers a variant of the original construction, one which yields a natural transverse affine structure for the foliation, and whose global holonomy lifts to an affine action of the group $\mathbb{Z}\left[\frac{1}{2}\right]$ on $\mathbb{R}$. Section 2 below describes the construction of the Hirsch foliation and some variations in codimension one.

The purpose of this note is to give a much broader generalization of the Hirsch construction to obtain foliations in codimension greater than one. It is possible that the constructions we describe, or some form of them, are "folklore" since the construction we give is very natural, but the authors do not know of any published reference for this construction.

Our construction is based on two observations, which can be developed in multiple ways. First, the Hirsch construction uses the classic solenoid embedding of the solid 2-torus into itself, where the core circle is mapped to itself by a $2-1$ map, which becomes the global holonomy of the resulting foliation. There is nothing special about the choice of a degree 2 map, and the construction is easily generalized to maps of degree $n$. More importantly, there is also nothing special about the use of a single self-embedding. The Hirsch construction generalizes to a collection of self-embeddings, and even further to realizing a given "system of étale correspondences" as the holonomy of a foliated compact manifold. The notion of a system of étale correspondences is introduced in Section 3, which generalizes that of a finitely-generated group.

The second observation about the Hirsch foliation is that the construction of the self-embedding uses the property of the circle $\mathbb{S}^{1}$ that it admits proper self-coverings. A manifold which admits no proper self-covering is said to be co-Hopfian. A group which admits no proper embedding into itself is said to be co-Hopfian. (The concept was introduced by R. Baer [2], and has been more recently studied by many authors; see Section 3.4.) The $q$-torus $\mathbb{T}^{q}$ is clearly not co-Hopfian, and for dimension $q \geq 3$ there are many more examples of manifolds which do admit proper self-coverings. All such examples give rise to foliations via a generalization of the Hirsch construction. For example, one obtains in this way a large collection of foliations of codimension $q$ whose transverse geometry is modeled on affine 
manifolds of dimension $q$, and the holonomy is generated by expanding diffeomorphisms. To illustrate the usefulness of this construction, we give three types of examples in Section 6, which hopefully convince the reader that these foliations often have very interesting dynamical properties.

Example 6.1 shows how to realize a class of Markov minimal sets using a a very simple construction. The result is a codimension one foliation whose holonomy has a unique exceptional minimal set with prescribed holonomy.

Example 6.3 constructs a smooth codimension two foliation which admits an exceptional minimal set that is homeomorphic to a Sierpinski 2torus. This provides an affirmative solution to problem 4 of [7]. More generally, the generalized Hirsch construction yields smooth foliations in arbitrary codimension with minimal sets which are transversally of the form of a Sierpinski manifold. This is discussed at length in the paper [5].

Example 6.4 constructs a foliation of codimension $q$, whose holonomy is locally equivalent to the action of the group of integer matrices $\mathbf{S L}(q, \mathbb{Z})$, but the foliation is not defined by an action of $\mathbf{S L}(q, \mathbb{Z})$ on $\mathbb{T}^{q}$. This is just one of many possible examples of this type.

The last Section 7 discusses some of the questions and problems suggested by these examples.

\section{Hirsch foliations in codimension one}

The "Hirsch example" is not just one example, but is rather a construction with two ingredients whose choices determine the properties of the resulting foliation. The original construction as in Hirsch [36] yields a real analytic foliation with an exceptional minimal set. On the other hand, the construction defined on pages 371-373 of [10] yields a minimal foliation which is transversally affine. We present here these constructions in full detail.

\subsection{Traditional construction}

The traditional construction of the affine Hirsch example proceeds as follows. Choose an analytic embedding of $S^{1}$ in the solid torus $D^{2} \times S^{1}$ so that its image is twice a generator of the fundamental group of the solid torus. See Figure 1 below.

Remove an open tubular neighborhood of the embedded $S^{1}$. What remains is a three dimensional manifold $N_{1}$ whose boundary is two disjoint copies of $T^{2}$. $D^{2} \times S^{1}$ fibers over $S^{1}$ with fibers the 2-disc. This fibration restricted to $N_{1}$ foliates $N_{1}$ with leaves consisting of 2-disks with two open subdisks removed. 
Now identify the two components of the boundary of $N_{1}$ by a diffeomorphism which covers the map $z \mapsto z^{2}$ of $S^{1}$ to obtain the manifold $N$. Endow $N$ with a Riemannian metric; then the punctured 2-disks foliating $N_{1}$ can now be viewed as pairs of pants.

As the foliation of $N_{1}$ is transverse to the boundary, the punctured 2disks assemble to yield a foliation of foliation $\mathcal{F}$ on $N$, where the leaves without holonomy (corresponding to irrational points for the chosen doubling map of $S^{1}$ ) are infinitely branching surfaces, decomposable into pairsof-pants which correspond to the punctured disks in $N_{1}$.

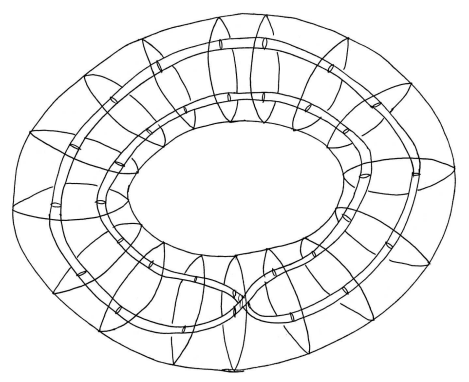

Figure 1. Original Hirsch construction illustrated

\subsection{General construction}

In this following, we give a more general construction of the Hirsch foliation in codimension one, which was described in the third author's thesis [55]. We ask the reader's patience for the discussion below; the reason is not to make the traditional construction more "obvious", but rather to explicitly list each of the steps which we will discuss later in the generalizations.

The first ingredient needed for the construction is the choice of an integer $n>1$, and an (analytic) embedding of $S^{1}$ in the solid torus $\mathbb{S}^{1} \times \mathbb{D}^{2}$ so that its image is $n$-times a generator of the fundamental group of the solid torus. Here is an explicit procedure for making this choice. Denote by

$$
\begin{aligned}
& \mathbb{D}^{2}=\{w \in \mathbb{C}|| w \mid \leq 1\} \subset \mathbb{C}, \\
& \mathbb{S}^{1}=\{w \in \mathbb{C}|| w \mid=1\} \subset \mathbb{D}^{2} .
\end{aligned}
$$

For $z \in \mathbb{C}$ with $0<|z|<1$ and $\epsilon>0$ such that $0<\epsilon<|z|$, set

$$
\begin{aligned}
& \mathbf{B}^{2}(z, \epsilon)=\{w \in \mathbb{C}|| w-z \mid<\epsilon\} \subset \mathbb{D}^{2}, \\
& \mathbf{S}^{1}(z, \epsilon)=\{w \in \mathbb{C}|| w-z \mid=\epsilon\} \subset \mathbb{D}^{2} .
\end{aligned}
$$


Set $\rho=e^{2 \pi \sqrt{-1} / n}$ which is a generator of the $n^{t h}$-roots of unity. Introduce the flat bundle

$$
\mathbb{E}=\left(\mathbb{R}^{1} \times \mathbb{R}^{2} /(x+1, z) \sim(x, \rho z)\right) \rightarrow \mathbb{S}^{1}
$$

which corresponds to the representation $\mathbb{Z} \rightarrow S O(2) \cong \mathbb{S}^{1}, n \mapsto \rho^{n}$. The unit disk subbundle of $\mathbb{E}$ is the "twisted" solid torus $N_{0}=\mathbb{R}^{1} \times \mathbb{D}^{2} /(x+$ $1, z) \sim(x, \rho z)$.

The flat bundle $\mathbb{E} \rightarrow \mathbb{S}^{1}$ is trivial as a vector bundle, with the bundle isomorphism $\mathbb{S}^{1} \times \mathbb{C} \cong \mathbb{E}$ induced by the map

$$
\begin{aligned}
& \widetilde{\Phi}: \mathbb{R} \times \mathbb{D}^{2} \rightarrow \mathbb{R} \times \mathbb{D}^{2}, \\
& \widetilde{\Phi}:(x, z) \rightarrow\left(x, e^{-2 \pi x \sqrt{-1} / n} z\right) .
\end{aligned}
$$

Note that

$$
\begin{aligned}
\widetilde{\Phi}(x+1, z)= & \left(x+1, e^{-2 \pi(x+1) \sqrt{-1} / n} z\right) \\
& =\left(x+1, \rho^{-1} e^{-2 \pi x \sqrt{-1} / n} z\right) \sim\left(x, e^{-2 \pi x \sqrt{-1} / n} z\right)=\widetilde{\Phi}(x, z)
\end{aligned}
$$

so that $\widetilde{\Phi}$ descends to a map $\Phi: \mathbb{S}^{1} \times \mathbb{C} \rightarrow \mathbb{E}$. The restriction also defines a trivialization of the unit disk bundles, again denoted by $\Phi: \mathbb{S}^{1} \times \mathbb{D}^{2} \rightarrow N_{0}$.

Now fix $z_{0} \in \mathbb{D}^{2}$ with $0<\left|z_{0}\right|<1$. For $0 \leq m<n$, set $z_{m}=\rho^{m} z_{0}$. Choose $\epsilon>0$ such that $2 \epsilon<\min \left\{\left|z_{0}\right|, 1-\left|z_{0}\right|\right\}$.

Define the punctured disk $\mathbf{P}_{0}^{2}$ obtained from $\mathbb{D}^{2}$ by deleting the $n$ disjoint open disks:

$$
\mathbf{P}_{0}^{2}=\mathbb{D}^{2}-\left(\mathbf{B}^{2}\left(z_{0}, \epsilon\right) \cup \mathbf{B}^{2}\left(z_{1}, \epsilon\right) \cup \cdots \cup \mathbf{B}^{2}\left(z_{n-1}, \epsilon\right)\right) .
$$

The result is illustrated in Figure 2 below.

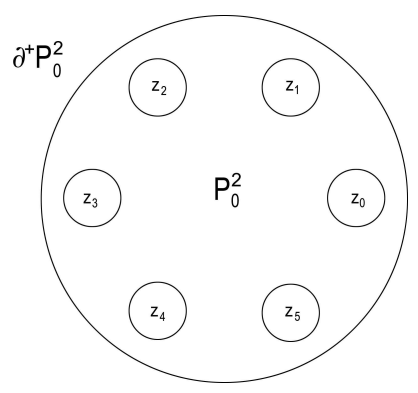

Figure 2. Basic pair of pants $\mathbf{P}_{0}^{2}$ with six legs 
Next, we introduce the 3-manifold $N_{1} \subset N_{0}$ with boundary as a quotient of $\mathbb{R}^{1} \times \mathbf{P}_{0}^{2}$

$$
N_{1}=\mathbb{R}^{1} \times \mathbf{P}_{0}^{2} /(x+1, z) \sim(x, \rho z) .
$$

Note that $N_{1}$ is diffeomorphic to the solid torus $\mathbb{S}^{1} \times \mathbb{D}^{2}$ with an open tubular neighborhood removed from an embedding of $\mathbb{S}^{1} \hookrightarrow \mathbb{S}^{1} \times \mathbb{D}^{2}$ which winds $n$-times around the core. The diffeomorphism is given by the restriction of the map $\Phi^{-1}: N_{0} \rightarrow \mathbb{S}^{1} \times \mathbb{D}^{2}$.

The boundary of $N_{1}$ consists of two disjoint tori, $\partial N_{1}=\partial^{+} N_{1} \cup \partial^{-} N_{1}$ where

$$
\begin{aligned}
& \partial^{+} N_{1}=\mathbb{R}^{1} \times \mathbb{S}^{1} /(x+1, z) \sim(x, \rho z), \\
& \partial^{-} N_{1}=\mathbb{R}^{1} \times\left(\mathbf{S}^{1}\left(z_{0}, \epsilon\right) \cup \cdots \cup \mathbf{S}^{1}\left(z_{n-1}, \epsilon\right)\right) /(x+1, z) \sim(x, \rho z) .
\end{aligned}
$$

There is a foliation $\mathcal{F}_{N_{1}}$ of $N_{1}$ whose leaves $\mathbf{P}_{x}^{2}$ are compact 2-manifolds with boundary, where:

$$
\begin{aligned}
\mathbf{P}_{x}^{2} & =\{x\} \times \mathbf{P}_{0}^{2} \subset N_{1}, \\
\mathbf{S}_{x}^{1} & =\{x\} \times \mathbb{S}^{1} \subset N_{1}, \\
\mathbf{S}_{x}^{1}\left(z_{i}, \epsilon\right) & =\{x\} \times \mathbf{S}^{1}\left(z_{i}, \epsilon\right) \subset \mathbf{P}_{x}^{2} .
\end{aligned}
$$

Note that the intersection of the leaf $\mathbf{P}_{x}^{2}$ with the boundary tori $\partial^{+} N_{1}$ and $\partial^{-} N_{1}$ consists of the circles $\mathbf{S}_{x}^{1}$ and $\mathbf{S}_{x}^{1}\left(z_{i}, \epsilon\right)$, so that each boundary torus is foliated by circles.

The second ingredient in the construction is the choice of a diffeomorphism $f: \partial^{+} N_{1} \rightarrow \partial^{-} N_{1}$ chosen so that $f$ maps the foliations of the boundary tori each to the other. Again, we give an explicit construction for $f$.

Choose an immersion $H: \mathbb{S}^{1} \rightarrow \mathbb{S}^{1}$ of degree $n$. The choice of $H$ is equivalent to the choice of a diffeomorphism $h: \mathbb{R} \rightarrow \mathbb{R}$ such that $h(x+1)=$ $h(x)+n$, and then $H=h \bmod (1)$.

Define an embedding $\widetilde{g}_{n}: \mathbb{R}^{1} \times \mathbb{D}^{2} \rightarrow \mathbb{R}^{1} \times \mathbb{D}^{2}$ by

$$
\widetilde{g}_{n}(x, z)=\left(h(x), e^{2 \pi h(x) \sqrt{-1} / n}\left(z_{1}+\epsilon z\right)\right)
$$

Then

$$
\begin{aligned}
& \widetilde{g}(x+1, z)=\left(h(x)+n, e^{2 \pi(h(x)+n) \sqrt{-1} / n}\left(z_{1}+\epsilon z\right)\right) \\
& \sim\left(h(x), e^{2 \pi h(x) \sqrt{-1} / n}\left(z_{1}+\epsilon z\right)\right)=\widetilde{g}(x, z)
\end{aligned}
$$

so that $\widetilde{g}$ induces an embedding $g: \mathbb{S}^{1} \times \mathbb{D}^{2} \rightarrow \mathbb{S}^{1} \times \mathbb{D}^{2}$ of the standard solid torus into itself. To obtain a map in terms of the twisted torus $N_{1}$, 
we conjugate $\widetilde{g}$ with $\widetilde{\Phi}$ of (1) to obtain $\tilde{f}: \mathbb{R}^{1} \times \mathbb{D}^{2} \rightarrow \mathbb{R}^{1} \times \mathbb{D}^{2}$ where $\widetilde{f}=\widetilde{\Phi} \circ \widetilde{g} \circ \widetilde{\Phi}^{-1}$. In coordinates,

$$
\begin{aligned}
\widetilde{f}(x, z) & =\widetilde{\Phi} \circ \widetilde{g}\left(x, e^{2 \pi x \sqrt{-1} / n} z\right) \\
& =\widetilde{\Phi}\left(h(x), e^{2 \pi h(x) \sqrt{-1} / n}\left(z_{1}+\epsilon e^{2 \pi x \sqrt{-1} / n} z\right)\right) \\
& =\left(h(x), e^{-2 \pi h(x) \sqrt{-1} / n} e^{2 \pi h(x) \sqrt{-1} / n}\left(z_{1}+\epsilon e^{2 \pi x \sqrt{-1} / n} z\right)\right) \\
& =\left(h(x),\left(z_{1}+\epsilon e^{2 \pi x \sqrt{-1} / n} z\right)\right) .
\end{aligned}
$$

Then

$$
\begin{aligned}
\widetilde{f}(x+1, z) & =\left(h(x)+n,\left(z_{1}+\epsilon e^{2 \pi(x+1) \sqrt{-1} / n} z\right)\right) \\
& =\left(h(x)+n,\left(z_{1}+\epsilon e^{2 \pi x \sqrt{-1} / n} \rho z\right)\right) \\
& \sim\left(h(x),\left(z_{1}+\epsilon e^{2 \pi x \sqrt{-1} / n} \rho z\right)\right) \\
& =\widetilde{f}(x, \rho z)
\end{aligned}
$$

so that $\tilde{f}$ descends to a map $f: N_{0} \rightarrow N_{0}$. By construction, the restriction of $\widetilde{f}$ defines a map $\widetilde{f}: \mathbb{R}^{1} \times \partial^{+} \mathbf{P}_{0}^{2} \rightarrow \mathbb{R}^{1} \times \partial^{-} \mathbf{P}_{0}^{2}$. It follows that $f$ induces a quotient map $f: \partial^{+} N_{1} \rightarrow \partial^{-} N_{1}$ which maps the outer boundary $\partial^{+} \mathbf{P}_{0}^{2}$ to the inner boundary $\partial^{-} \mathbf{P}_{0}^{2}$. Define

$$
N=N_{1} /(x, z) \sim f(x, z) .
$$

Note that $\tilde{f}$ maps fibers to fibers, so the leaves of $\mathcal{F}_{N_{1}} \cap \partial^{+} N_{1}$ are mapped to leaves of $\mathcal{F}_{N_{1}} \cap \partial^{-} N_{1}$, hence $N$ has a foliation $\mathcal{F}$ whose leaves are the unions of $n$-punctured disks $\mathbf{P}_{x}^{2}$.

\subsection{Description of leaves}

The typical leaf of $\mathcal{F}$ is modeled on a homogeneous $n$-partite tree, though exceptional leaves of $\mathcal{F}$ contain isolated handles. Let $0 \leq x<1$ and consider the $n$-punctured disk $\mathbf{P}_{x}^{2} \subset N_{1}$. The inner boundary consists of $n$ disjoint circles,

$$
\partial^{-} \mathbf{P}_{x}^{2}=\mathbf{S}_{x}^{1}\left(z_{0}, \epsilon\right) \cup \cdots \cup \mathbf{S}_{x}^{1}\left(z_{n-1}, \epsilon\right) .
$$

The map $H: \mathbb{S}^{1} \rightarrow \mathbb{S}^{1}$ is a submersion of degree $n$, so the set $H^{-1}(x)=$ $\left\{x_{0}, \ldots, x_{n-1}\right\}$ consists of $n$ distinct points. The map $f$ identifies the outer boundary circle $\mathbf{S}_{x_{\ell}}^{1}=\partial^{+} \mathbf{P}_{x_{\ell}}^{2}$ with an inner boundary component of $\partial^{-} \mathbf{P}_{x}^{2}$, $f: \mathbf{S}_{x_{\ell}}^{1} \rightarrow \mathbf{S}_{x}^{1}\left(z_{i}, \epsilon\right)$ for some $i=i(\ell)$. Note that the identification joins the outer circle to the inner circle rotated by the amount $\rho^{i}$.

This processes is iterated both in reverse and forward times, to yield the leaf $L_{x}$ through $x$. Figure 3 illustrates the case $n=2$, where $\mathbf{P}_{0}^{2}$ is a 
two-punctured disk. Note that the rotation in joining the outer and inner boundary circles is by multiples of -1 , so is not apparent in the illustration.

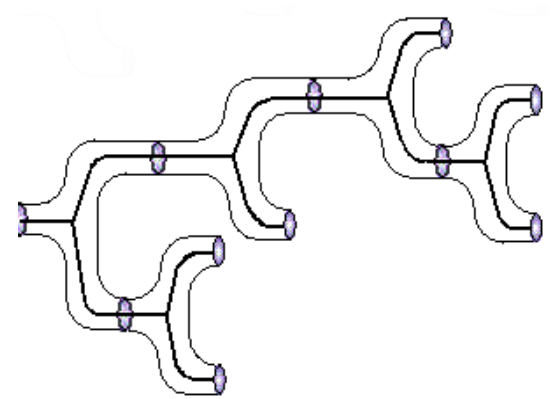

Figure 3. Typical leaf

In the exceptional case where $x$ is a fixed-point for $H$, then $x \in H^{-1}(x)$, so we assume $x=x_{0}$. Then the outer boundary circle $\partial^{+} \mathbf{P}_{x}^{2}$ is identified with an inner boundary circle of $\partial^{-} \mathbf{P}_{x}^{2}$. Thus, the identifications used to construct the leaf $L_{x}$ "in the future" all collapse into a circular identification on the punctured surface $\mathbf{P}_{x}^{2}$ which creates a handle on $L_{x}$ with a closed loop that generates transverse holonomy for $\mathcal{F}$. The leaf $L_{x}$ is modeled on a pointed $n$-partite tree, with a terminal vertex corresponding to the closed loop produced by the fixed point $H\left(x_{0}\right)=x_{0}$.

\subsection{Transverse holonomy}

The foliation $\mathcal{F}$ on $N$ admits a complete transversal, $T: \mathbb{S}^{1} \hookrightarrow N$, constructed as follows: the origin $0 \in \mathbf{P}_{0}^{2}$ so we can define an embedding $\widetilde{t}: \mathbb{R} \rightarrow \mathbb{R}^{1} \times \mathbf{P}_{0}^{2}$ where $\widetilde{t}(x)=(x, 0)$. Then

$$
\widetilde{t}(x+1)=(x+1,0) \sim(x, \rho \cdot 0)=(x, 0)=\widetilde{t}(x) .
$$

Passing to quotient manifolds we obtain $t: \mathbb{S}^{1} \rightarrow N_{1}$. Clearly, the image of $t$ intersects each leaf of $\mathcal{F}_{N_{1}}$ and thus descends to a complete transversal for the foliation $\mathcal{F}$ on $N$, denoted by $T: \mathbb{S}^{1} \rightarrow N$. We will let $\mathbb{S}_{T}^{1}$ denote the image of this map, which is identified with $\mathbb{S}^{1}$.

Next, consider the holonomy transformations induced on the transversal $\mathbb{S}_{T}^{1}$ by $\mathcal{F}$. The foliation $\mathcal{F}_{N_{1}}$ is defined by a fibration, so has no holonomy. Thus, all of the holonomy of $\mathcal{F}$ is induced by the identification of the outer and inner boundaries via the map $H$. One can visualize this holonomy 
action by considering a short interval $(a, b) \subset \mathbb{S}_{T}^{1}$, considered as in interval in the covering $\mathbb{R}^{1}$, and then sliding it across the leaves of $N_{1}$, avoiding the holes removed on the inner boundary, until reaching the outer boundary $\partial^{+} \mathbf{N}_{1}$. Apply the map $h$ to the points in the interval $(a, b)$ to obtain the interval $(h(a), h(b))$ which is identified with an interval in one of the inner boundary components $\partial^{-} N_{1}$. Then slide the interval $(h(a), h(b))$ along the leaves of $\mathcal{F}_{N_{1}}$ back to the transversal $\mathbb{S}_{T}^{1}$.

Note that this holonomy construction requires that the domain interval $(a, b)$ is not a closed loop, as otherwise the sliding actions demanded above cannot be performed. The image of the full transversal $\mathbb{S}_{T}^{1}$ cannot be parallel transported past the interior boundary of $N_{1}$ as the inner core links the embedded torus. This is the basis of the remarkable property of the Hirsch foliation, that even though $\mathcal{F}$ has a complete closed transversal, the foliation is not equivalent to a group action on that transversal. The map $H$ is not invertible.

\subsection{Affine Hirsch foliation}

The affine Hirsch foliation is obtained by choosing an integer $n>1$ and setting $h(x)=n x$. Clearly, $h(x+1)=h(x)+n$. Moreover, the transverse holonomy as described above is obviously affine, as the map $x \mapsto n x$ is an affine transformation.

We consider one other aspect of this example, the existence of leaves with holonomy for $\mathcal{F}$. Transverse holonomy for $\mathcal{F}$ arises exactly from periodic orbits of $H: \mathbb{S}^{1} \rightarrow \mathbb{S}^{1}$. We use the modular notation for $H$ so that $H(x)=n x(1)$. Then $0 \leq x<1$ is a fixed point for some power $H^{k}$ if and only if $n^{k} x=x(1)$. Thus, $x=\ell /\left(n^{k}-1\right)$ for some integer $0 \leq \ell<n^{k}-1$. Each such point then generates a closed loop in the leaf $L_{x}$ through $x$ with non-trivial transverse holonomy.

Note that the set of points $\mathcal{P}=\left\{x=\ell /\left(n^{k}-1\right) \mid k \geq 1,0 \leq \ell<\right.$ $\left.n^{k}-1\right\} \subset \mathbb{S}^{1}$ is dense, so $\mathcal{F}$ has a dense set of leaves with non-trivial holonomy.

\subsection{Hirsch foliation with exceptional minimal set}

The construction given by Hirsch in [36] includes an explicit description of the map $H: \mathbb{S}^{1} \rightarrow \mathbb{S}^{1}$ of degree 2 . Define $H$ in terms of the map $h:[0,1] \rightarrow$ $[0,2]$ illustrated in Figure 4, and defined by

$$
\begin{gathered}
h(0)=0 ; h(.5)=1.5 ; h(.75)=1.75 ; h(1)=2 ; h^{\prime}(.75)<1, \\
h(x)>3 x \& h^{\prime}(x)>1 \text { for } 0<x<.5 .
\end{gathered}
$$




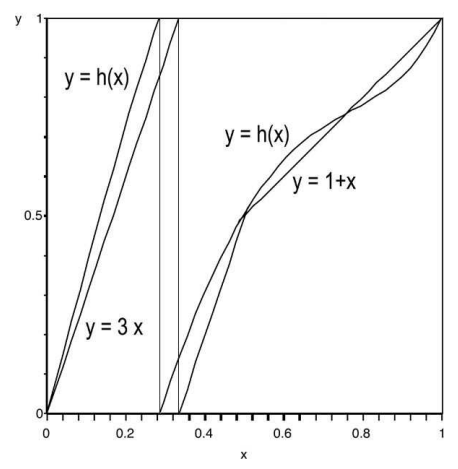

Figure 4. 2-1 map with structurally stable fixed-point

Two points $x, y \in \mathbb{S}^{1}$ are said to be in the same "grand orbit" of $H$ if there are positive integers $k, \ell$ such that $H^{k}(x)=H^{\ell}(y)$ (cf. Milnor [48].) This defines an equivalence relation on $\mathbb{S}^{1}$. We need only check the transitive condition: suppose $H^{k}(x)=H^{\ell}(y)$ and $H^{u}(y)=H^{v}(z)$, then note $H^{u+k}(x)=H^{u+\ell}(y)=H^{\ell+v}(z)$.

Let $\mathcal{O}(x) \subset \mathbb{S}^{1}$ denote all the points in the same orbit as $x$. A subset $\mathbf{K} \subset \mathbb{S}^{1}$ is $H$-invariant if for all $x \in \mathbf{K}$, the orbit $\mathcal{O}(x) \subset \mathbf{K}$. The set $\mathbf{K}$ is minimal if $\mathbf{K}$ is closed, and for all $x \in \mathbf{K}$ the orbit $\mathcal{O}(x)$ is dense in $\mathbf{K}$. A minimal set $\mathbf{K}$ is exceptional if it is nowhere dense and not a finite set.

Lemma 2.1 Let $H: \mathbb{S}^{1} \rightarrow \mathbb{S}^{1}$ be defined by the map in Figure 4. Then there exists a unique minimal set $\mathbf{K} \subset \mathbb{S}^{1}$.

Proof. Define the intervals

$$
\begin{gathered}
\mathcal{I}=[0, .5] \subset \mathbb{R}^{1} / x \sim x+1 \cong \mathbb{S}^{1}, \\
\mathcal{J}=(.5,1) \subset \mathbb{R}^{1} / x \sim x+1 \cong \mathbb{S}^{1} .
\end{gathered}
$$

The point $z_{0}=.75 \in \mathcal{J}$ is a fixed by $H$, and the open interval $\mathcal{J}$ is a basin of attraction for $y_{0}$.

Define the open, $H$-invariant set $U=\bigcup_{w \in \mathcal{J}} \mathcal{O}(w)$.

Set $\mathbf{K}=\mathbb{S}^{1}-U$, which is a closed invariant subset of $\mathcal{I}$. The boundary points for $x_{0}=0$ and $y_{0}=.5$ for $\mathcal{I}$ are fixed-points for $H$, so $\mathcal{O}\left(x_{0}\right) \subset \mathbf{K}$ and $\mathcal{O}\left(y_{0}\right) \subset \mathbf{K}$.

The property $h^{\prime}(x) \geq x$ on $\mathcal{I}$ implies that $h$ is expansive on $\mathcal{I}$, hence for any $x_{0} \leq a<b \leq y_{0}$, there exists $\ell>0$ such that $h^{\ell}(a, b) \cap \mathcal{J} \neq \emptyset$. Hence 
$U \cap \mathcal{I}$ is dense in $\mathcal{I}$ and thus $\mathbf{K}$ is nowhere dense.

We must show that for $x \in \mathbf{K}$, the grand orbit $\mathcal{O}(x)$ is dense in $\mathbf{K}$. Note that $\mathcal{K}=\overline{\mathcal{O}\left(x_{0}\right)} \subset \mathbf{K}$. Given $x \in \mathbf{K} \subset \mathcal{I}$, as $h:[0, .5) \rightarrow[0,1.5)$ is expansive, the grand orbit $\mathcal{O}(x)$ contains the sequence of points $\left\{h^{-\ell}(x) \mid \ell=1,2, \ldots\right\}$ which converge to $x_{0}$ and thus $x_{0} \in \overline{\mathcal{O}(x)}$. This implies that $\mathcal{K} \subset \overline{\mathcal{O}(x)} \subset \mathbf{K}$. Hence, it suffices to show that $\mathcal{K}=\mathbf{K}$, or that for every $x \in \mathbf{K}$ there is a point in $\mathcal{O}\left(x_{0}\right)$ arbitrarily close.

Let $x \in \mathbf{K}$ and $\epsilon>0$, then the intersection $(x-\epsilon, x+\epsilon) \cap U \neq \emptyset$. Choose $z \in(x-\epsilon, x+\epsilon) \cap U$.

Let $(a, b) \subset U$ be the largest interval such that $a<z<b$. Then either $a \in(x-\epsilon, x+\epsilon)$ or $b \in(x-\epsilon, x+\epsilon)$. Otherwise, we have that $(x-\epsilon, x+\epsilon) \subset(a, b) \subset U$, which contradicts $x \notin U$.

The point $z \in U$ implies there is some $w \in \mathcal{J}$ such that $H^{k}(z)=H^{\ell}(w)$, and as $H: \mathcal{J} \rightarrow \mathcal{J}$ is the basin of attraction for $z_{0}$ we have $H^{k}(z) \in \mathcal{J}$. As $H^{-k}(\mathcal{J}) \subset U$ by definition, there is a connected component $\mathcal{J}_{1} \subset H^{-k}(\mathcal{J})$ which contains $z$. Then $\mathcal{J}_{1} \cap(a, b) \neq \emptyset$ and $(a, b)$ maximal implies $\mathcal{J}_{1} \subset$ $(a, b)$.

The endpoints of $\mathcal{J}$ are $y_{0}=.5$ and $x_{0}=1$, hence the endpoints of $\mathcal{J}_{1}$ are contained in the orbits $\mathcal{O}\left(y_{0}\right)$ and $\mathcal{O}\left(x_{0}\right)$. As $x_{0}, y_{0} \in \mathbf{K}$, it follows that $\mathcal{J}_{1}=(a, b)$ where $H^{k}(a)=y_{0}$ and $H^{k}(b)=x_{0}$. This is exactly what one expects in analogy with the construction of the usual Cantor set, that the gaps in $\mathbb{S}^{1}-\mathbf{K}$ consists of the maximal connected components in the wandering domain, which in this case is $U$.

If $b \in(x-\epsilon, x+\epsilon)$ then $\mathcal{O}\left(x_{0}\right) \cap(x-\epsilon, x+\epsilon) \neq \emptyset$.

If $a \in(x-\epsilon, x+\epsilon)$, we need the observation that $y_{0} \in \overline{\mathcal{O}\left(x_{0}\right)}$, hence $\mathcal{O}\left(x_{0}\right)$ intersects every open neighborhood of every point in $\mathcal{O}\left(y_{0}\right)$ which implies $\mathcal{O}\left(x_{0}\right) \cap(x-\epsilon, x+\epsilon) \neq \emptyset$.

To show that $y_{0} \in \overline{\mathcal{O}\left(x_{0}\right)}$ note that $y_{1}=h^{-1}(1)>0$, and that $y_{2}=$ $h^{-1}\left(1+y_{1}\right)>y_{1}$. In general, by induction we have that $y_{n+1}=h^{-1}(1+$ $\left.y_{n}\right)>y_{n}$ and the sequence $\left\{y_{n}\right\}$ is monotonically increasing to $y_{0}=1 / 2$.

\section{$3 \quad$ Systems of étale correspondences}

The suspension of a smooth action of a finitely generated group $\Gamma$ on a compact manifold $M$ without boundary is one of the main methods of constructing foliations cited in textbooks $[9,10,23,33]$. The basic idea is to choose a set of generators $\left\{\gamma_{1}, \ldots, \gamma_{k}\right\}$ for $\Gamma$ so that for each $1 \leq i \leq k$ there is a diffeomorphism $h_{i}=h\left(\gamma_{i}\right): M \rightarrow M$. The second step is to choose $k$ pairs of disjoint disks in the 2 -sphere $\mathbb{S}^{2}$, label the pairs $\left(\mathbb{D}_{i}^{s}, \mathbb{D}_{i}^{r}\right)$ 
and chose a diffeomorphism of the boundaries $\phi_{i}: \partial \mathbb{D}_{i}^{s} \rightarrow \partial \mathbb{D}_{i}^{r}$. Then the manifold

$$
N_{1}=M \times\left(\mathbb{S}^{2}-\mathbb{D}_{1}^{s}-\mathbb{D}_{1}^{r}-\cdots-\mathbb{D}_{k}^{s}-\mathbb{D}_{k}^{r}\right)
$$

has a foliation $\mathcal{F}_{0}$ defined by the projection to the first factor $M$. Moreover, $N_{1}$ has $2 k$ boundary components, each diffeomorphic to $M \times \mathbb{S}^{1}$. The restriction of $\mathcal{F}_{0}$ to each boundary component is given by the circle fibers. The boundary components are then pairwise identified by the maps $h_{i} \times$ $\phi_{i}: M \times \partial \mathbb{D}_{i}^{s} \rightarrow M \times \partial \mathbb{D}_{i}^{r}$ to obtain a compact foliated manifold $N$ with $M$ as transversal, and global holonomy equivalent to the action of $\Gamma$ on $M$.

The codimension one Hirsch construction is analogous to the above suspension construction, except that there is a single holonomy map $h: \mathbb{S}^{1} \rightarrow$ $\mathbb{S}^{1}$ which is a covering map, but not a diffeomorphism. Our generalization of this construction, given in Sections 4 and 5, gives a method to realize a foliation whose holonomy is generated by a collection of endomorphisms of a given compact manifold $M$, to form what we call here a system of étale correspondences. The generating endomorphisms need not be coverings, but are only required to be local covering maps, hence the notation "étale". The generating maps are diffeomorphisms of appropriate covering spaces of $M$.

Let $M$ be an oriented compact manifold without boundary of dimension $q$. We assume that there is a Riemannian metric on $T M$ such that for $\omega$ the volume form on $M$ associated to the Riemannian metric and the orientation of $M$, then $M$ has total volume 1 . The Riemannian metric yields a norm on each tangent space $T_{x} M$, which we denote by $\|\cdot\|_{x}$.

\subsection{Correspondences}

An étale correspondence for $M$ is a triple of data $(s, r, h)=(s: P \rightarrow$ $M, r: Q \rightarrow M, h: P \rightarrow Q)$ where

- $s: P \rightarrow M$ is a covering map of finite index $m$ which is a local isometry;

- $r: Q \rightarrow M$ is a covering map of finite index $n$ which is a local isometry;

- $h: P \rightarrow Q$ is a diffeomorphism.

We say that $(s, r, h)$ is a correspondence of type $(m, n)$. The data yields a diagram 


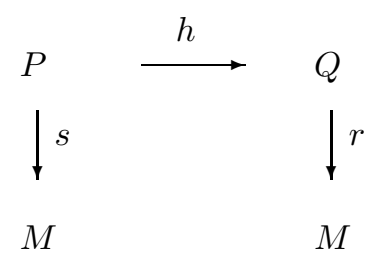

For example, if $M$ is simply connected, then every covering map of $M$ is a diffeomorphism, so the maps $s$ and $r$ are necessarily isometric diffeomorphisms, and an étale correspondence is essentially just a choice of a diffeomorphism $r \circ h \circ s^{-1}: M \rightarrow M$.

The simplest non-trivial example is for the case $M=\mathbb{S}^{1}$ with metric such that $\mathbb{S}^{1}$ has total length 1 . For a positive integer $n$, let $\times_{n}: \mathbb{S}^{1} \rightarrow \mathbb{S}^{1}$ denote the covering map $z \mapsto z^{n}$. Given a pair of positive integers $m, n$ we take $P=Q=\mathbb{S}^{1}, s=\times_{m}$ and $r=\times_{n}$. Note that for the lifted Riemannian metrics, $P$ has total length $m$ and $Q$ has total length $n$. A diffeomorphism $h: P \rightarrow Q$ yields an étale correspondence. The special case $m=1, n>1$ was considered in Section 2, for in this case the composition $H=r \circ h \circ s^{-1}: \mathbb{S}^{1} \rightarrow \mathbb{S}^{1}$ is an immersion of degree $n$.

\subsection{Expansive maps}

An orientation-preserving immersion $f: M \rightarrow M$ is expanding if there exists $C>1$ such that

$$
\|d f(x)(\vec{v})\|_{h(x)} \geq C \cdot\|\vec{v}\|_{x} \text { for all } \vec{v} \in T_{x} M .
$$

Let $n=\operatorname{deg}(f) \geq 1$ be the topological degree of $f$. Let $[\omega] \in H^{q}(M ; \mathbb{R})$ denote the cohomology class of the closed form $\omega$. Then $\left[f^{*} \omega\right]=f^{*}[\omega]=$ $n \cdot[\omega]$, so that

$$
n=\int_{M} f^{*} \omega>C^{q} \cdot \int_{M} \omega=C^{q} \cdot 1>1 .
$$

An immersion is a local covering map, and since $M$ is compact, it follows that $f: M \rightarrow M$ is a covering map of degree $n>1$. Chose a basepoint $x_{0} \in M$ and let $y_{0} \in M$ be such that $f\left(y_{0}\right)=x_{0}$. Then the induced map on fundamental groups, $f_{\#}: \pi_{1}\left(M, y_{0}\right) \rightarrow \pi_{1}\left(M, x_{0}\right)$, has image a proper subgroup $\Pi_{f} \subset \pi_{1}\left(M, x_{0}\right)$.

Let $r: Q \rightarrow M$ be a canonical covering associated to the subgroup $\Pi_{f} \subset \pi_{1}\left(M, x_{0}\right)$. (Say, the covering defined by the path-space construction.) Endow $Q$ with the lifted Riemannian metric so that the covering map $r$ is a local isometry. Then the total volume of $Q$ is $n$. 
Let $h: M \rightarrow Q$ be a lift of the map $f$, so that $r \circ h=f$. Then $h$ is an immersion of degree 1 , hence a diffeomorphism. Thus, take $P=M$ and let $s: P \rightarrow M$ be the identity, and we obtain an étale correspondence $(s, r, h)$.

The existence of an expanding map $f: M \rightarrow M$ is a very strong hypothesis on $M$. It implies that the universal cover $\widetilde{M} \rightarrow M$ has polynomial volume growth rate $[35,56]$, and hence by Gromov [27] the fundamental group $\pi_{1}\left(M, x_{0}\right)$ has a nilpotent subgroup of finite index. The most obvious example is for $M=\mathbb{T}^{q}$, but there are many further examples where $\pi_{1}\left(M, x_{0}\right)$ is a non-abelian nilpotent group. For example, Hyunkoo Lee and Kyung-Bai Lee prove in [45] that every nilmanifold whose fundamental group is two-step nilpotent admits an expanding map. This result was generalized by Karel Dekimpe and Kyung-Bai Lee, who gave a criteria for a nilmanifold that it admit an expanding map in [14], and they classified those nilpotent Lie algebras which admit expanding maps in [15].

\subsection{Products}

Given two étale correspondences

$$
\begin{aligned}
& \left(s_{1}: P_{1} \rightarrow M_{1}, r_{1}: Q_{1} \rightarrow M_{1}, h_{1}: P_{1} \rightarrow Q_{1}\right), \\
& \left(s_{2}: P_{2} \rightarrow M_{2}, r_{2}: Q_{2} \rightarrow M_{2}, h_{2}: P_{2} \rightarrow Q_{2}\right),
\end{aligned}
$$

we can form the product correspondence

$$
(s: P \rightarrow M, r: Q \rightarrow M, h: P \rightarrow Q),
$$

where $M=M_{1} \times M_{2}$ with the product metric, $s=s_{1} \times s_{2}, r=r_{1} \times r_{2}$, and $h=h_{1} \times h_{2}$. For example, let $\left(s_{1}, r_{1}, h_{1}\right)$ be the étale correspondence associated to an expanding map $f_{1}: M_{1} \rightarrow M_{1}$. Let $f_{2}: M_{2} \rightarrow M_{2}$ be a diffeomorphism of a compact oriented Riemannian manifold, then let $P_{2}=Q_{2}=M_{2}$ with $s_{2}, r_{2}$ both the identity maps, and set $h_{2}=f_{2}$. Then the product map $f=\left(r_{1} \times\right.$ id $) \circ\left(h_{1} \times h_{2}\right): M_{1} \times M_{2} \rightarrow M_{1} \times M_{2}$ is a partially expanding map.

\subsection{Self-coverings and the co-Hopf condition}

A special case of an étale correspondence $(s, r, h)=(s: P \rightarrow M, r: Q \rightarrow$ $M, h: P \rightarrow Q)$ is when the source map $s: P \rightarrow M$ is a diffeomorphism, so $m=1$, and the range map $r: Q \rightarrow M$ has degree $n>1$. Then the composition $f=r \circ h \circ s^{-1}: M \rightarrow M$ is a proper self-covering. The fundamental group $\pi_{1}\left(M, x_{0}\right)$ must therefore be non-trivial, and the induced map $f_{\#}: \pi_{1}\left(M, x_{0}\right) \rightarrow \pi_{1}\left(M, x_{0}\right)$ is a proper self-embedding. Moreover, given a proper self-covering $f: M \rightarrow M$ and diffeomorphisms $g_{1}$ and $g_{2}$ of $M$, then 
$f_{1}=g_{1} \circ f \circ g_{2}$ is again a proper self-covering, so the existence of one such map ensures the existence of a wide variety of examples.

A group which admits no proper self-embedding is said to be co-Hopfian, a concept introduced by Reinholt Baer [2]. The existence of proper selfcoverings is related to the venerable question of which fundamental groups do not have the co-Hopfian property. Ohshika and Potyagailo [49] and Kapovich and Wise [44] discuss the history of the co-Hopfian property. Belegradek [3] gave a criterion for when a finitely generated torsion-free nilpotent group is co-Hopfian.

Note that while the fundamental group $\pi_{1}\left(M, x_{0}\right)$ of a closed manifold $M$ which admits proper self-coverings is not co-Hopfian, the converse is far from clear.

The $q$-torus $\mathbb{T}^{q}$ is the canonical example of a closed manifold admitting proper self-coverings. There are no other oriented examples for dimension $q=2$.

The study of which 3-manifold groups are co-Hopfian is formulated in terms of the eight geometries in the Thurston Geometrization Conjecture [57]. Clearly, $M=\Sigma \times \mathbb{S}^{1}$ where $\Sigma$ is a closed surface, admits proper self-coverings. The next simplest examples are when $M$ is a non-trivial Seifert fiber space over an orbifold. González-Acuña, Litherland and Whitten proved in [24] that if $M$ is a closed 3-dimensional Seifert fiber space, then its fundamental group is co-Hopfian, if and only if $M$ does not cover itself non-trivially, if and only if $M$ admits a geometric structure modeled on $S^{3}$ or on $\widetilde{\mathrm{SL}(2, \mathbb{R})}$. Thus, 5 of the 7 geometries which are Seifert fibered admit non-trivial self-coverings.

González-Acuña and Whitten studied which Haken manifolds have the co-Hopfian property in their paper [26]. The work of Shi-cheng Wang and Qing Wu [59] used the Gromov norm invariant [28, 57] of closed 3-manifolds to study the co-Hopf property; in particular, all hyperbolic 3-manifolds have non-zero Gromov norm, so are co-Hopfian. Leonid Potyagaŭlo and Shi Van (a.k.a. Shi-cheng Wang) study whether the fundamental group of a 3 -manifold satisfying Thurston's conjecture is a co-Hopfian group in [50], and obtain some necessary and sufficient conditions.

The study of connected sums of 3-manifolds leads to the study of the class of graph manifolds. Shi-cheng Wang and Feng-chun Yu studied in [61] the co-Hopfian property of graph manifolds. More generally, they considered the related Property $\mathrm{C}$ that, whenever $M_{1}, M_{2}$ are homeomorphic finite covering spaces of $M$, the degrees of the coverings are the same. They proved that a closed geometric 3-manifold $M$ has Property $\mathrm{C}$ if and only if $M$ is not covered by either $\Sigma \times \mathbb{S}^{1}$ or a torus bundle over $\mathbb{S}^{1}$. The sur- 
vey paper by Buyalo and Svetlov [8] also gives results on the co-Hopfian property for graph manifolds.

In dimension $q>3$, one class of closed manifolds which admit proper self-covering maps are those which admit an expanding map $f: M \rightarrow M$. As noted in Section 3.2, the fundamental group of $M$ necessarily has a nilpotent subgroup of finite index. It is natural to ask which finitely-generated nilpotent groups are co-Hopfian, or not. This problem was solved by Igor Belegradek in [3]. Examples of non-Hopfian nilpotent groups give rise to self-covering maps of nil-manifolds, which are typically partially expanding.

The co-Hopfian property has been studied for two other classes of finitely-generated groups. Ohshika and Potyagailo [49], Wang and Zhou [60], and Delzant and Potyagailo [17] study which Kleinian groups are co-Hopfian. Note that if a Kleinian group is torsion-free and co-compact, then the corresponding hyperbolic manifold $M$ has non-zero Gromov norm, so is coHopfian.

The question of which (word) hyperbolic groups are co-Hopfian was posed by Gromov and Thurston. Sela proved in [53] that a non-elementary, torsion-free hyperbolic group is co-Hopfian if and only if it is freely indecomposable. Later, Kapovich and Wise showed in [44] that the co-Hopf property does not typically descend to subgroups of word hyperbolic groups.

We mention two other results which concern the topological properties of spaces which admit proper self-coverings. Delgado and Timm [16] gives restrictions on the fundamental group of a connected finite complex that has nontrivial finite connected coverings. Andrica and Funar [1] give Morse type obstructions to the existence of homeomorphisms between coverings of a closed manifold.

\subsection{Systems of étale correspondences}

A system of étale correspondences for the Riemannian manifold $M$ is a collection

$$
\mathcal{C}=\left\{\left(s_{\ell}: P_{\ell} \rightarrow M, r_{\ell}: Q_{\ell} \rightarrow M, h_{\ell}: P_{\ell} \rightarrow Q_{\ell} \mid 1 \leq \ell \leq k\right\},\right.
$$

where each $\left(s_{\ell}, r_{\ell}, h_{\ell}\right)$ is an étale correspondence of type $\left(m_{\ell}, n_{\ell}\right)$.

Given a finitely generated group $\Gamma$ and a smooth action $\varphi: \Gamma \times M \rightarrow M$, choose a set of generators $\left\{\gamma_{1}, \ldots, \gamma_{k}\right\}$ for $\Gamma$ then set $P_{\ell}=Q_{\ell}=M$, let $s, r: M \rightarrow M$ be the identity maps, and $f_{\ell}=\varphi\left(\gamma_{\ell}\right)$. This yields a system of étale correspondences $\mathcal{C}$ for $M$, where $\left(s_{\ell}, r_{\ell}, h_{\ell}\right)=\left(\right.$ id, id, $\left.f_{\ell}\right)$ so $\left(m_{\ell}, n_{\ell}\right)=$ $(1,1)$.

Conversely, when all of the indices $n_{\ell}=m_{\ell}=1$ then for each $\ell$ we obtain a diffeomorphism $f_{\ell}=r_{\ell} \circ h_{\ell} \circ s_{\ell}^{-1}$, so that the system of étale 
correspondences yields a collection of diffeomorphisms $\left\{f_{1}, \ldots f_{k}\right\}$ which generate a subgroup $\Gamma \subset \operatorname{Diff}(M)$.

To analyze the general case, fix a basepoint $x_{0} \in M$, and set $\Pi=$ $\pi_{1}\left(M, x_{0}\right)$. Introduce the collection of all finite index subgroups of $\Pi$, denoted by $\Delta=\{\pi \subset \Pi \mid[\Pi: \pi]<\infty\}$. For each $\pi \in \Delta$ let $p_{\pi}: P_{\pi} \rightarrow M$ be the covering of $M$ associated to the model of the universal covering $\widetilde{M} \rightarrow M$, using paths based at $x_{0}$. (In other words, we fix a canonical model $p_{\pi}: P_{\pi} \rightarrow M$ for the covering associated to each $\pi$.) Give $P_{\pi}$ the Riemannian metric induced by the covering map $p_{\pi}$, so that $p_{\pi}$ is a local isometry.

We say that an étale correspondence $(s, r, h)$ is standard (with respect to these choices) if there are subgroups $\pi^{s}, \pi^{r} \in \Delta$ such that

$$
(s, r, h)=\left(p_{\pi^{s}}: P_{\pi^{s}} \rightarrow M, p_{\pi^{r}}: P_{\pi^{r}} \rightarrow M, h: P_{\pi^{s}} \rightarrow P_{\pi^{r}}\right) .
$$

The correspondence $(s, r, h)$ is said to have index $\left(\pi^{s}, \pi^{r}\right)$, so the type is $(n, m)$ where $m=\left[\Pi: \pi^{s}\right]$ and $n=\left[\Pi: \pi^{r}\right]$. Given two standard étale correspondences $\left(s_{1}, r_{1}, h_{1}\right)$ of type $\left(\pi_{1}^{s}, \pi_{1}^{r}\right)$ and $\left(s_{2}, r_{2}, h_{2}\right)$ of type $\left(\pi_{2}^{s}, \pi_{2}^{r}\right)$, if $\pi_{1}^{r}=\pi_{2}^{s}$ then we can compose then to obtain

$$
\begin{aligned}
& \left(s_{1}, r_{1}, h_{1}\right) \circ\left(s_{2}, r_{2}, h_{2}\right) \\
& \quad=\left(s_{1}: P_{\pi_{1}^{s}} \rightarrow M, r_{2}: P_{\pi_{2}^{r}} \rightarrow M, h_{2} \circ h_{1}: P_{\pi_{1}^{s}} \rightarrow P_{\pi_{2}^{r}}\right) .
\end{aligned}
$$

In this way, the standard étale correspondences form a pseudogroup $\mathcal{P}(\Delta)$ with object space the disjoint union

$$
\mathcal{P}=\bigcup_{\pi \in \Delta} P_{\pi} .
$$

When $M$ is simply connected, or more if generally $\Pi$ has no subgroups of finite index, then $\mathcal{P}(\Delta)=\operatorname{Diff}(\mathrm{M})$. If $\Pi$ does admit a subgroup $\pi \subset \Pi$ of finite index, then each $f \in \operatorname{Diff}(\mathrm{M})$ admits at least one lift to a diffeomorphism $h: P_{\pi} \rightarrow P_{\pi}$ so that $\mathcal{P}(\Delta)$ is no longer simply Diff(M).

The above construction is most interesting when the fundamental group $\Pi$ admits many subgroups of finite index; for example, when it is infinite and residually finite. In fact, we include the above discussion on composition of étale correspondences, because such a system gives rise to cohomology invariants, obtained from the geometric realization of the topological category $\mathcal{P}(\Delta)$. These cohomology invariants may help characterize the pseudogroup modeled on $M$ obtained from the étale correspondences modeled on $M$. 


\subsection{Correspondences and pseudogroups}

In general, a system of étale correspondences $\mathcal{C}=\left\{\left(s_{\ell}: P_{\ell} \rightarrow M, r_{\ell}: Q_{\ell} \rightarrow\right.\right.$ $\left.M, h_{\ell}: P_{\ell} \rightarrow Q_{\ell} \mid 1 \leq \ell \leq k\right\}$ for $M$ corresponds to a particular type of pseudogroup modeled on $M$. Let $\mathcal{U}$ denote the collection of all open subsets of $M$ which are contractible in $M$. For each $1 \leq \ell \leq k$, the covering map $s_{\ell}: P_{\ell} \rightarrow M$ has degree $m_{\ell}$, and for each $U \in \mathcal{U}$ the inverse image

$$
s_{\ell}^{-1}(U)=\left\{\widetilde{U}_{\ell, 1}, \ldots, \widetilde{U}_{\ell, m_{\ell}}\right\}
$$

consists of $m_{\ell}$ disjoint open connected subsets $\widetilde{U}_{\ell, i} \subset P_{\ell}$. For each $1 \leq i \leq$ $m_{\ell}$ the restriction $s_{\ell} \mid \widetilde{U}_{\ell, i} \rightarrow U$ is a diffeomorphism, so we can define the immersion

$$
h_{\ell, i, U}=r_{\ell} \circ h_{\ell} \circ\left(s_{\ell} \mid \widetilde{U}_{\ell, i}\right)^{-1}: U \rightarrow M .
$$

The collection of maps

$$
\Gamma_{\mathcal{C}}=\left\{h_{\ell, i, U} \mid 1 \leq \ell \leq k, 1 \leq i \leq m_{\ell}, U \in \mathcal{C}\right\}
$$

generates a compactly pseudogroup modeled on $M$ (cf. [29, 30,31]), which we again denote by $\Gamma_{\mathcal{C}}$.

One of the open questions in foliation theory, is which compactly supported pseudogroups can be realized as the pseudogroup of a foliation on a compact manifold without boundary. In Section 5 we use a more general form of the Hirsch construction to realize every pseudogroup $\Gamma_{\mathcal{C}}$ arising from a system of étale correspondences as the pseudogroup of a foliation.

\section{Generalized Hirsch foliations}

The generalization of the Hirsch construction of Section 2.2 will be given in two parts. In this section, we realize a single étale correspondence as the holonomy of a foliation. In the next section, we extend the construction to realize a given system of correspondences.

Let $(s, r, h)=\left(p_{\pi^{s}}: P_{\pi^{s}} \rightarrow M, p_{\pi^{r}}: P_{\pi^{r}} \rightarrow M, h: P_{\pi^{s}} \rightarrow P_{\pi^{r}}\right)$ be an étale correspondence in standard form with type $(m, n)$. The first step is the construction in Section 4.3 of the foliated manifold $N_{1}$ with boundary $\partial N_{1}=\partial^{s} N_{1} \cup \partial^{r} N_{1}$. We then use $h$ to define a foliation preserving diffeomorphism $H: \partial^{s} N_{1} \rightarrow \partial^{r} N_{1}$ which yields the foliated manifold $N$ via the identification of the boundary components.

The construction of the Hirsch foliation in codimension one in Section 2.2 begins with the choice of a point $0 \neq z_{0} \in \mathbb{D}^{2}$ and we form the set $z_{m}=\rho^{m} z_{0}$, where $\rho$ is an $n^{t h}$ root of unity. The set $\left\{z_{0}, z_{1}, \ldots z_{n-1}\right\}$ is the orbit of a cyclic subgroup of $\mathbf{O}(2)$ of order $n$ acting on $\mathbb{D}^{2}$. These 
points are the centers of the disks removed in order to obtain $\mathbf{P}_{0}^{2}$. The crucial observation in the generalization of the Hirsch construction is to replace the cyclic group acting on $\mathbb{D}^{2}$ with a finite subgroup of the orthogonal group $\mathbf{O}(p+1)$ acting on the unit sphere $\mathbb{S}^{p}$, where $p$ depends upon the structure of the correspondence. The $n$-punctured 2-disk $\mathbf{P}_{0}^{2}$, which can be viewed as an $n+1$-punctured 2-sphere, will accordingly be replaced with a suitably punctured $p$-sphere, so the leaves of the foliation we obtain will have dimension $p$.

\subsection{Flat bundles}

Recall that $\Pi=\pi_{1}\left(M, x_{0}\right)$. Define the finite coset spaces $X^{s}=\Pi / \pi^{s}$ and $X^{r}=\Pi / \pi^{r}$. Note that we do not assume the subgroups $\pi^{s}$ and $\pi^{r}$ are normal in $\Pi$, so these coset spaces are not necessarily groups. They do, however, inherit a left action of $\Pi$, which acts as a group of permutations on each $X^{s}$ and $X^{r}$. Let $\mu^{s}: \Pi \rightarrow \operatorname{Perm}\left(X^{s}\right)$ and $\mu^{r}: \Pi \rightarrow \operatorname{Perm}\left(X^{r}\right)$ be the corresponding representations.

Let $m$ denote the cardinality of $X^{s}$, and $n$ that of $X^{r}$.

Let $\mathbb{V}^{s}=\mathbb{R}\left\langle X^{s}\right\rangle$ denote the inner product $\mathbb{R}$-vector space with orthonormal basis $\left\{\vec{u}_{g} \mid g \in X^{s}\right\}$.

The permutation action $\mu^{s}$ of $\Pi$ on $X^{s}$ induces a representation $\rho^{s}: \Pi \rightarrow$ $\operatorname{Aut}\left(\mathbb{V}^{s}\right) \cong \mathbf{O}(m)$.

Similarly define the space $\mathbb{V}^{r}=\mathbb{R}\left\langle X^{r}\right\rangle$ with orthonormal basis $\left\{\vec{v}_{g} \mid g \in\right.$ $\left.X^{r}\right\}$, and induced representation $\rho^{r}: \Pi \rightarrow \operatorname{Aut}\left(\mathbb{V}^{r}\right) \cong \mathbf{O}(n)$.

Let $\mathbb{V}=\mathbb{V}^{s} \oplus \mathbb{V}^{r}$ be the orthogonal direct sum, with orthonormal basis $\left\{\vec{u}_{g} \mid g \in X^{s}\right\} \cup\left\{\vec{v}_{g} \mid g \in X^{r}\right\}$. Let $\rho=\rho^{s} \times \rho^{r}: \Pi \rightarrow \mathbf{O}(m) \times \mathbf{O}(n) \subset$ $\mathbf{O}(m+n)$ be the product representation.

Define a flat vector bundle over $M$ by

$$
\mathbb{E}=\widetilde{M} \times \mathbb{V} /\{(\gamma \cdot x, \vec{v}) \sim(x, \rho(\gamma) \vec{v}), \forall \gamma \in \Pi\} \rightarrow M
$$

where $\widetilde{M} \rightarrow M$ is the universal covering of $M$, and $\Pi$ acts on the left on $\widetilde{M}$ by deck transformations. Note that the representation $\rho$ induces an action of $\Pi$ on $\mathbb{V}$ by isometries, so $\mathbb{E}$ inherits a fiberwise inner product from the inner product on $\mathbb{V}$. Let $\mathbb{E}_{1} \subset \mathbb{E}$ denote the subbundle of unit vectors, so if we let $\mathbb{V}_{1} \subset \mathbb{V}$ denote the unit vectors in $\mathbb{V}$, then

$$
\mathbb{E}_{1}=\widetilde{M} \times \mathbb{V}_{1} /\{(\gamma \cdot x, \vec{v}) \sim(x, \rho(\gamma) \vec{v}), \forall \gamma \in \Pi\} \rightarrow M .
$$

The bundle $\mathbb{E} \rightarrow M$ need not be trivial, even though $\mathbb{E}$ is flat. However, as $M$ is paracompact, there exists a vector bundle $\mathbb{F} \rightarrow M$ such that the direct sum $\mathbb{E} \oplus \mathbb{F} \rightarrow M$ is the trivial bundle. Choose such a bundle $\mathbb{F}$ with fiber dimension $\xi$, give $\mathbb{F}$ a fiberwise inner product, and give $\mathbb{E} \oplus \mathbb{F}$ the 
direct sum inner product. Let $\varepsilon^{p}=M \times \mathbb{R}^{p}$ denote the product bundle, where $p=m+n+\xi$, endowed with the standard the fiberwise inner product inherited from the standard metric on $\mathbb{R}$. Fix a bundle isomorphism $\Phi: \mathbb{E} \oplus$ $\mathbb{F} \cong \varepsilon^{p}$ which is a fiberwise isometric map. Finally, let $\varepsilon^{p+1}=\varepsilon^{p} \oplus \varepsilon$ be the orthogonal direct sum, where the additional summand of the trivial line bundle $\varepsilon$ is also given the fiberwise inner product inherited from the standard metric on $\mathbb{R}$.

Let $N_{0}=M \times \mathbb{S}^{p} \subset \varepsilon^{p+1}$ denote the $\mathbb{S}^{p}$-subbundle of unit vectors in $\varepsilon^{p+1}$.

\subsection{Tubular sections}

The next step in the construction is to define submanifolds $W_{0}^{s}, W_{0}^{r} \subset N_{0}$ of dimension $q$ such that the projection $N_{0} \rightarrow M$ restricts to covering maps associated with the subgroups $\pi^{s}$ and $\pi^{r}$, respectively. (Recall that $q$ is the dimension of $M$.) We will first construct submanifolds $W^{s}, W^{r} \subset \mathbb{E}_{1}$ such that the projection $\mathbb{E}_{1} \rightarrow M$ restricts to the required covering maps, and then use the inclusion followed by the trivialization map $\Phi$ to obtain the isometric embedding

$$
\iota_{0}: \mathbb{E}_{1} \subset \mathbb{E} \subset \mathbb{E} \oplus \mathbb{F} \oplus \varepsilon \cong \varepsilon^{p+1}
$$

to obtain $W_{0}^{s}$ and $W_{0}^{r}$. The stabilizing summands $\mathbb{F} \oplus \varepsilon$ have no role in the construction of $W^{s}$ and $W^{r}$, but are rather introduced so that for $\epsilon>0$ sufficiently small, the normal $\epsilon$-disk bundles of the submanifolds $W_{0}^{s}, W_{0}^{r} \subset N_{0}$ are trivial.

Let $1^{s} \in X^{s}$ denote the coset $\left[\pi^{s}\right] \in X^{s}$, and similarly define $1^{r} \in X^{r}$. Let $\overrightarrow{1}^{s} \in \mathbb{V}^{s} \subset \mathbb{V}^{s} \oplus \mathbb{V}^{r}=\mathbb{V}$ be the basis element corresponding to the coset $1^{s}$, and $\overrightarrow{1}^{r} \in \mathbb{V}^{r} \subset \mathbb{V}^{s} \oplus \mathbb{V}^{r}=\mathbb{V}$ be the basis element corresponding to $1^{r}$.

For $\gamma \in \Pi$, set $z_{\gamma}=\rho(\gamma)\left(\overrightarrow{1}^{s}\right) \in \mathbb{V}_{1}$ and $w_{\gamma}=\rho(\gamma)\left(\overrightarrow{1}^{r}\right) \in \mathbb{V}_{1}$. We let $z_{0}=\overrightarrow{1}^{s}$ and $w_{0}=\overrightarrow{1}^{r}$.

Note that if $\delta \in \pi^{s}$ then $z_{\delta}=z_{0}$, and more generally $z_{\gamma \delta}=z_{\gamma}$. Thus, for each coset $g \in X^{s}=\Pi / \pi^{s}$ there is a well-defined point $z_{g} \in \mathbb{V}_{1}$. Of course, $z_{g}$ is just the point on the sphere $\mathbb{V}_{1}$ corresponding to the basis vector $\vec{u}_{g}$.

Likewise, if $\delta \in \pi^{r}$ then $w_{\delta}=w_{0}$, and more generally $w_{\gamma \delta}=w_{\gamma}$. Thus, for each coset $g \in X^{r}=\Pi / \pi^{r}$ there is a well-defined point $w_{g} \in \mathbb{V}_{1}$ which corresponds to the basis vector $\vec{v}_{g}$.

Set $\mathcal{O}^{s}=\left\{z_{g} \mid g \in X^{s}\right\}$ and $\mathcal{O}^{r}=\left\{w_{g} \mid g \in X^{r}\right\}$. Note that both sets are invariant under the action of $\rho$. Define submanifolds of $\mathbb{E}_{1}$ by

$$
\begin{aligned}
& W^{s}=\widetilde{M} \times \mathcal{O}^{s} /\{(\gamma \cdot x, \vec{v}) \sim(x, \rho(\gamma) \vec{v}), \quad \forall \gamma \in \Pi\}, \\
& W^{r}=\widetilde{M} \times \mathcal{O}^{r} /\{(\gamma \cdot x, \vec{v}) \sim(x, \rho(\gamma) \vec{v}), \quad \forall \gamma \in \Pi\} .
\end{aligned}
$$


Since the action of $\Pi$ on $\mathcal{O}^{s}$ is transitive with stabilizer group $\pi^{s}$, the projection $\mathbb{E}_{1} \rightarrow M$ restricted to the manifold $W^{s}$ is the standard covering of $M$ associated to the subgroup $\pi^{s}$.

Similarly, the action of $\Pi$ on $\mathcal{O}^{r}$ is transitive with stabilizer group $\pi^{r}$, hence the projection $\mathbb{E}_{1} \rightarrow M$ restricted to the manifold $W^{r}$ is the standard covering of $M$ associated to the subgroup $\pi^{r}$.

Each fiber of $\mathbb{E}_{1} \rightarrow M$ over $x \in M$ is naturally isometric to the unit sphere $\mathbb{S}^{m+n-1} \subset \mathbb{V}$, and is given the induced Riemannian metric with geodesic distance function $d_{x}^{v}$, so has circumference $2 \pi$. Given any pair of orthogonal unit vectors $\vec{v}, \vec{u} \in \mathbb{V}$, we have $d_{x}^{v}(\vec{v}, \vec{u})=\pi / 2>1$.

The submanifolds $W^{s}$ and $W^{r}$ intersect the fiber of $\mathbb{E}_{1} \rightarrow M$ over $x$ in points corresponding to the orbits $\mathcal{O}^{s}$ and $\mathcal{O}^{r}$. Thus, for distinct points $z, w \in \mathcal{O}^{s} \cup \mathcal{O}^{r}$, the distance $d_{\mathbb{S} p}(z, w)=\pi / 2>1$.

Define $W_{0}^{s}, W_{0}^{r} \subset N_{0}$ as the images of $W^{s}$ and $W^{r}$ respectively under the map $\iota_{0}$ of (11), so we obtain diffeomorphisms $\iota_{0}^{s}: W^{s} \rightarrow W_{0}^{s}$ and $\iota_{0}^{r}: W^{r} \rightarrow W_{0}^{r}$.

Let $\vec{n}_{0}: M \rightarrow N_{0}$ be the section defined by $\vec{n}_{0}(x)=\{x\} \times(0, \ldots, 0,1)$. Similarly, let $\vec{s}_{0}: M \rightarrow N_{0}$ be the section defined by $\vec{s}_{0}(x)=\{x\} \times$ $(0, \ldots, 0,-1)$. The section $\vec{n}_{0}$ should be viewed as determining the "north pole" for each $\mathbb{S}^{p}$-fiber of $N_{0} \rightarrow M$, and $\vec{s}_{0}$ is the opposite "south pole". The manifold $N_{0}$ with this section deleted is

$$
N_{0}-\vec{n}_{0}(M)=M \times\left\{\mathbb{S}^{p}-(0, \ldots, 0,1)\right\} \cong M \times \mathbb{R}^{p},
$$

where the last isomorphism uses stereographic projection from the south pole in each fiber. For each $z \in N_{0}-\vec{n}_{0}(M)$ the identification (12) induces a framing of the fiberwise tangent space $T_{z}^{v} N_{0}$ of $N_{0}$ at $z$.

Each fiber $\{x\} \times \mathbb{S}^{p}$ of $N_{0}=M \times \mathbb{S}^{p}$ over $x \in M$ has the standard Riemannian metric with geodesic distance function denoted by $d_{\mathbb{S}^{p}}$, and with circumference $2 \pi$. The inclusion $\iota_{0}: \mathbb{E}_{1} \rightarrow N_{0}$ is a fiberwise isometric embedding, and the image of $\iota_{0}$ is fiberwise orthogonal to the section $\vec{n}$. Hence, for each $x \in M$, the submanifolds $W_{0}^{s}$ and $W_{0}^{r}$ intersect the fiber of $N_{0} \rightarrow M$ over $x$ in points which are fiberwise orthogonal to $\vec{n}(x)$. Let $W_{x}^{s}=W_{0}^{s} \cap\left(\{x\} \times \mathbb{S}^{p}\right)$ and $W_{x}^{r}=W_{0}^{r} \cap\left(\{x\} \times \mathbb{S}^{p}\right)$. Then for each point $z \in W_{x}^{s}$ or $W_{x}^{r}$ the fiberwise distance to the north pole $\vec{n}_{0}(x)$ is $\pi / 2$.

Let $0<\epsilon<\pi / 4$, then each $x \in M$ we define the fiberwise disk neighborhoods of $W_{x}^{s}$ and $W_{x}^{r}$ by

$$
\begin{aligned}
& \mathbb{D}^{p}\left(W_{x}^{s}, \epsilon\right)=\bigcup_{w \in W_{x}^{s}}\left\{(x, z) \in M \times \mathbb{S}^{p} \mid d_{\mathbb{S}^{p}}(z, w)<\epsilon\right\} \subset\{x\} \times \mathbb{S}^{p}, \\
& \mathbb{D}^{p}\left(W_{x}^{r}, \epsilon\right)=\bigcup_{w \in W_{x}^{r}}\left\{(x, z) \in M \times \mathbb{S}^{p} \mid d_{\mathbb{S}^{p}}(z, w)<\epsilon\right\} \subset\{x\} \times \mathbb{S}^{p},
\end{aligned}
$$


and their boundaries

$$
\begin{aligned}
& \mathbb{S}^{p-1}\left(W_{x}^{s}, \epsilon\right)=\bigcup_{w \in W_{x}^{s}}\left\{(x, z) \in M \times \mathbb{S}^{p} \mid d_{\mathbb{S}^{p}}(z, w)=\epsilon\right\} \subset\{x\} \times \mathbb{S}^{p}, \\
& \mathbb{S}^{p-1}\left(W_{x}^{r}, \epsilon\right)=\bigcup_{w \in W_{x}^{r}}\left\{(x, z) \in M \times \mathbb{S}^{p} \mid d_{\mathbb{S}^{p}}(z, w)=\epsilon\right\} \subset\{x\} \times \mathbb{S}^{p} .
\end{aligned}
$$

Fix $\epsilon=1 / 10$, then define the open tubular neighborhoods of $W_{0}^{s}$ and $W_{0}^{r}$ in $N_{0}$ by

$$
\begin{aligned}
& \mathbb{D}^{p}\left(W_{0}^{s}\right)=\bigcup_{x \in M} \mathbb{D}^{p}\left(W_{x}^{s}, 1 / 10\right), \\
& \mathbb{D}^{p}\left(W_{0}^{r}\right)=\bigcup_{x \in M} \mathbb{D}^{p}\left(W_{x}^{r}, 1 / 10\right),
\end{aligned}
$$

and their boundaries in $N_{0}$ by

$$
\begin{aligned}
& T^{s}=\bigcup_{x \in M} \mathbb{S}^{p-1}\left(W_{x}^{s}, 1 / 10\right), \\
& T^{r}=\bigcup_{x \in M} \mathbb{S}^{p-1}\left(W_{x}^{r}, 1 / 10\right) .
\end{aligned}
$$

\subsection{Construction of the foliation}

We are now prepared to complete the construction. Set $N_{1}=N_{0}-$ $\left(\mathbb{D}^{p}\left(W_{0}^{s}\right) \cup \mathbb{D}^{p}\left(W_{0}^{r}\right)\right)$.

The boundary of $N_{1}$ has two connected components, $\partial N_{1}=\partial^{s} N_{1} \cup$ $\partial^{r} N_{1}$, where $\partial^{s} N_{1}=T^{s}$ and $\partial^{r} N_{1}=T^{r}$. The manifold $N_{1}$ fibers over $M$, defining a foliation $\mathcal{F}_{0}$. The fiber of $N_{1}$ over $x \in M$ is the set

$$
\mathbf{P}_{x}^{p}=N_{1} \cap\left(\{x\} \times \mathbb{S}^{p}\right)=\left(\{x\} \times \mathbb{S}^{p}\right)-\left(\mathbb{D}^{p}\left(W_{x}^{s}, 1 / 10\right) \cup \mathbb{D}^{p}\left(W_{x}^{r}, \epsilon\right)\right),
$$

so that the typical leaf of $\mathcal{F}_{0}$ is diffeomorphic to the sphere $\mathbb{S}^{p}$ with $m+n$ disks removed.

Whereas the traditional "pair of pants" $\mathbf{P}_{0}^{2}$ used in Section 2.2 has one hole considered as its "waist", and has $n$ holes for the "legs", this modern hosiery represented by $\mathbf{P}_{x}^{p}$ has $m$ waist holes and $n$ leg holes. Moreover, it has dimension $p=m+n+\xi$.

The submanifold $T^{s}$ is disjoint from the north pole section $\vec{n}_{0}$ so the fibers of the map $T^{s} \rightarrow M$ are trivialized by the map (12). The similar statement holds for $T^{r}$, so we obtain fiberwise identifications

$$
\begin{aligned}
& \varphi^{s}: T^{s} \cong W_{0}^{s} \times \mathbb{S}^{p-1}, \\
& \varphi^{r}: T^{r} \cong W_{0}^{r} \times \mathbb{S}^{p-1} .
\end{aligned}
$$


Finally, we are given the diffeomorphism $h: P_{\pi^{s}} \rightarrow P_{\pi^{r}}$ where $P_{\pi^{s}}$ is standard, so canonically identified with $W^{s}$ and hence with $W_{0}^{s}$, while $P_{\pi^{r}}$ is identified with $W_{0}^{r}$. Thus, $h$ induces a diffeomorphism

$$
H=\left(\varphi^{r}\right)^{-1} \circ(h \times \mathrm{Id}) \circ \varphi^{s}: T^{s} \rightarrow T^{r},
$$

which maps fibers to fibers. That is, $H$ preserves the foliation on the boundary components of $N_{1}$ induced by $\mathcal{F}_{0}$.

Define $N=N_{1} / T^{s} \sim T^{r}$, and let $\mathcal{F}$ be the foliation whose leaves are composed of the images under the identification map $H$ of the leaves of $\mathcal{F}_{0}$. This completes the construction of the "Hirsch foliation" $\mathcal{F}$ on $N$ realizing the étale correspondence $(s, r, h)$.

\subsection{Remarks on the construction}

The boundary manifolds $T^{s}$ and $T^{r}$ are sphere bundles over the covering spaces $W_{0}^{s} \cong P_{\pi^{s}} \rightarrow M$ and $W_{0}^{r} \cong P_{\pi^{r}} \rightarrow M$, but due to the fact that the flat bundle $\mathbb{E} \rightarrow M$ may have very complicated structure, and the trivialization $\mathbb{E} \oplus \mathbb{F}$ of this bundle is given abstractly, the embedding of these manifolds into $M \times \mathbb{S}^{p}$ is not easily described. In fact, every aspect of the above construction is more technically complicated, but the overall construction is exactly analogous.

The manifold $M$ has a natural embedding $M_{0}=\vec{s}(M)$ into $N$ as the image of the south pole section of $M \times \mathbb{S}^{p}$. The proof that the holonomy pseudogroup of $\mathcal{F}$ induced on $M_{0}$ is equivalent to that defined by the étale correspondence $(s, r, h)$ on $M$ is also analogous to the proof for the traditional Hirsch foliation. Hence, the dynamics of $\mathcal{F}$ induced on the section $M_{0}$ is equivalent to the dynamics of $h$ "acting" on $M$.

For each $x \in M \cong M_{0}$, the leaf $L_{x}$ of $\mathcal{F}$ through $x$ is assembled from a countable collection of leaves $\mathbf{P}_{y}^{p}$ of $\mathcal{F}_{0}$,

$$
L_{x}=\bigcup_{y \sim x} \mathbf{P}_{y}^{p} / \sim
$$

where $y \sim x$ means that they are on the same orbit of $x \in M$ under the étale correspondence $h$.

It would be quite complicated to try to describe the exact geometry of the leaves and their embeddings into $N$, as the identification of the various boundary spheres of the building blocks $\mathbf{P}_{y}^{p}$ uses the map $H$, whose fiberwise component reflects the topology of the flat bundle $\mathbb{E}$ and its trivialization. It is an interesting question whether there is in fact some topological invariant of $\mathcal{F}$ reflected by the geometry of the embeddings of the leaves. For example, Heitsch and Hurder calculated the foliated coarse cohomology of 
the traditional Hirsch foliation (with holonomy $h(z)=z^{2}$ ) in the paper [34]. It would be quite interesting to understand the foliated coarse cohomology of the Hirsch foliation $\mathcal{F}$ corresponding to an étale correspondence $(s, r, h)$, and whether the coarse cohomology depends upon the topology of the embeddings of the leaves into $N$.

\section{$5 \quad$ Realizing systems of étale correspondences}

Suppose there is given a system of étale correspondences for the Riemannian manifold $M$

$$
\mathcal{C}=\left\{\left(s_{\ell}: P_{\ell} \rightarrow M, r_{\ell}: Q_{\ell} \rightarrow M, h_{\ell}: P_{\ell} \rightarrow Q_{\ell}\right) \mid 1 \leq \ell \leq k\right\},
$$

where each $\left(s_{\ell}, r_{\ell}, h_{\ell}\right)$ is an étale correspondence of type $\left(m_{\ell}, n_{\ell}\right)$ and index $\left(\pi_{\ell}^{s}, \pi_{\ell}^{r}\right)$. In this section, we show how to modify the construction of the last section to realize the system $\mathcal{C}$ as the holonomy of a foliation $\mathcal{F}$.

\subsection{Flat bundles}

For each $1 \leq \ell \leq k$, define the finite coset spaces $X_{\ell}^{s}=\Pi / \pi_{\ell}^{s}$ and $X_{\ell}^{r}=$ $\Pi / \pi_{\ell}^{r}$ with left action of $\Pi$ by permutations. Let $\mu_{\ell}^{s}: \Pi \rightarrow \operatorname{Perm}\left(X_{\ell}^{s}\right)$ and $\mu_{\ell}^{r}: \Pi \rightarrow \operatorname{Perm}\left(X_{\ell}^{r}\right)$ be the corresponding permutation representations.

Let $m_{\ell}$ denote the cardinality of $X_{\ell}^{s}$, and $n_{\ell}$ that of $X_{\ell}^{r}$.

Let $\mathbb{V}_{\ell}^{s}=\mathbb{R}\left\langle X_{\ell}^{s}\right\rangle$ denote the inner product $\mathbb{R}$-vector space with orthonormal basis $\left\{\vec{u}_{\ell, g} \mid g \in X_{\ell}^{s}\right\}$.

The permutation action $\mu_{\ell}^{s}$ of $\Pi$ on $X_{\ell}^{s}$ induces a representation $\rho_{\ell}^{s}: \Pi \rightarrow$ $\operatorname{Aut}\left(\mathbb{V}_{\ell}^{s}\right) \cong \mathbf{O}\left(m_{\ell}\right)$.

Similarly define the space $\mathbb{V}_{\ell}^{r}=\mathbb{R}\left\langle X_{\ell}^{r}\right\rangle$ with orthonormal basis $\left\{\vec{v}_{\ell, g} \mid\right.$ $\left.g \in X_{\ell}^{r}\right\}$, and induced representation $\rho_{\ell}^{r}: \Pi \rightarrow \operatorname{Aut}\left(\mathbb{V}_{\ell}^{r}\right) \cong \mathbf{O}\left(n_{\ell}\right)$.

basis

Let $\mathbb{V}=\bigoplus_{\ell=1}^{k} \mathbb{V}_{\ell}^{s} \oplus \mathbb{V}_{\ell}^{r}$ be the orthogonal direct sum, with orthonormal

$$
\mathcal{S}=\bigcup_{\ell=1}^{k}\left\{\vec{u}_{\ell, g} \mid g \in X^{s}\right\} \cup\left\{\vec{v}_{\ell, g} \mid g \in X^{r}\right\} .
$$

Set $m=m_{1}+\cdots+m_{k}$ and $n=n_{1}+\cdots+n_{k}$. Let

$$
\begin{aligned}
\rho=\rho_{1}^{s} \times \rho_{1}^{r} \times & \cdots \times \rho_{k}^{s} \times \rho_{k}^{r}: \\
\Pi & \rightarrow \mathbf{O}\left(m_{1}\right) \times \mathbf{O}\left(n_{1}\right) \times \cdots \times \mathbf{O}\left(m_{k}\right) \times \mathbf{O}\left(n_{k}\right) \subset \mathbf{O}(m+n)
\end{aligned}
$$

be the product representation. 
The rest of the construction proceeds almost exactly as for a single étale correspondence. Define a flat vector bundle over $M$ by

$$
\mathbb{E}=\widetilde{M} \times \mathbb{V} /\{(\gamma \cdot x, \vec{v}) \sim(x, \rho(\gamma) \vec{v}), \forall \gamma \in \Pi\} \rightarrow M,
$$

where $\widetilde{M} \rightarrow M$ is the universal covering of $M$, and $\Pi$ acts on the left on $\widetilde{M}$ by deck transformations. The representation $\rho$ induces an action of $\Pi$ on $\mathbb{V}$ by isometries, so $\mathbb{E}$ inherits a fiberwise inner product from the inner product on $\mathbb{V}$. Let $\mathbb{E}_{1} \subset \mathbb{E}$ denote the subbundle of unit vectors, and let $\mathbb{V}_{1} \subset \mathbb{V}$ denote the unit vectors in $\mathbb{V}$, then

$$
\mathbb{E}_{1}=\widetilde{M} \times \mathbb{V}_{1} /\{(\gamma \cdot x, \vec{v}) \sim(x, \rho(\gamma) \vec{v}), \forall \gamma \in \Pi\} \rightarrow M .
$$

There exists a vector bundle $\mathbb{F} \rightarrow M$ such that the direct sum $\mathbb{E} \oplus \mathbb{F} \rightarrow M$ is the trivial bundle. Choose such a bundle $\mathbb{F}$ with fiber dimension $\xi$, give $\mathbb{F}$ a fiberwise inner product, and give $\mathbb{E} \oplus \mathbb{F}$ the direct sum inner product. Let $\varepsilon^{p}=M \times \mathbb{R}^{p}$ denote the product bundle, where $p=m+n+\xi$, endowed with the standard the fiberwise inner product inherited from the standard metric on $\mathbb{R}$. Fix a bundle isomorphism $\Phi: \mathbb{E} \oplus \mathbb{F} \cong \varepsilon^{p}$ which is a fiberwise isometric map. Finally, let $\varepsilon^{p+1}=\varepsilon^{p} \oplus \varepsilon$ be the orthogonal direct sum, where the additional summand of the trivial line bundle $\varepsilon$ is also given the fiberwise inner product inherited from the standard metric on $\mathbb{R}$.

Let $N_{0}=M \times \mathbb{S}^{p} \subset \varepsilon^{p+1}$ denote the $\mathbb{S}^{p}$-subbundle of unit vectors in $\varepsilon^{p+1}$.

\subsection{Tubular sections}

Let $1_{\ell}^{s} \in X_{\ell}^{s}$ denote the coset $\left[\pi_{\ell}^{s}\right] \in X_{\ell}^{s}$, and similarly define $1_{\ell}^{r} \in X_{\ell}^{r}$. Let $\overrightarrow{1}_{\ell}^{s} \in \mathbb{V}_{\ell}^{s} \subset \mathbb{V}$ be the basis element corresponding to the coset $1_{\ell}^{s}$, and $\overrightarrow{1}_{\ell}^{r} \in \mathbb{V}_{\ell}^{r} \subset \mathbb{V}$ be the basis element corresponding to $1_{\ell}^{r}$.

For $\gamma \in \Pi$, set $z_{\ell, \gamma}=\rho(\gamma)\left(\overrightarrow{1}_{\ell}^{s}\right) \in \mathbb{V}_{1}$ and $w_{\ell, \gamma}=\rho(\gamma)\left(\overrightarrow{1}_{\ell}^{r}\right) \in \mathbb{V}_{1}$. We let $z_{\ell, 0}=\overrightarrow{1}_{\ell}^{s}$ and $w_{\ell, 0}=\overrightarrow{1}_{\ell}^{r}$.

Note that if $\delta \in \pi_{\ell}^{s}$ then $z_{\ell, \delta}=z_{\ell, 0}$, and more generally $z_{\ell, \gamma \delta}=z_{\ell, \gamma}$. Thus, for each coset $g \in X_{\ell}^{s}=\Pi / \pi_{\ell}^{s}$ there is a well-defined point $z_{\ell, g} \in \mathbb{V}_{1}$. Of course, $z_{\ell, g}$ is just the point on the sphere $\mathbb{V}_{1}$ corresponding to the basis vector $\vec{u}_{\ell, g}$.

Likewise, if $\delta \in \pi_{\ell}^{r}$ then $w_{\ell, \delta}=w_{\ell, 0}$, and more generally $w_{\ell, \gamma \delta}=w_{\ell, \gamma}$. Thus, for each coset $g \in X_{\ell}^{r}=\Pi / \pi_{\ell}^{r}$ there is a well-defined point $w_{\ell, g} \in \mathbb{V}_{1}$ which corresponds to the basis vector $\vec{v}_{\ell, g}$.

Set $\mathcal{O}_{\ell}^{s}=\left\{z_{\ell, g} \mid g \in X_{\ell}^{s}\right\}$ and $\mathcal{O}_{\ell}^{r}=\left\{w_{\ell, g} \mid g \in X_{\ell}^{r}\right\}$. Note that both sets are invariant under the action of $\rho$. For $1 \leq \ell \leq k$, define submanifolds 
of $\mathbb{E}_{1}$ by

$$
\begin{aligned}
& W_{\ell}^{s}=\widetilde{M} \times \mathcal{O}_{\ell}^{s} /\{(\gamma \cdot x, \vec{v}) \sim(x, \rho(\gamma) \vec{v}), \forall \gamma \in \Pi\}, \\
& W_{\ell}^{r}=\widetilde{M} \times \mathcal{O}_{\ell}^{r} /\{(\gamma \cdot x, \vec{v}) \sim(x, \rho(\gamma) \vec{v}), \quad \forall \gamma \in \Pi\} .
\end{aligned}
$$

Since the action of $\Pi$ on $\mathcal{O}_{\ell}^{s}$ is transitive with stabilizer group $\pi_{\ell}^{s}$, the projection $\mathbb{E}_{1} \rightarrow M$ restricted to the manifold $W_{\ell}^{s}$ is the standard covering of $M$ associated to the subgroup $\pi_{\ell}^{s}$.

Similarly, the action of $\Pi$ on $\mathcal{O}_{\ell}^{r}$ is transitive with stabilizer group $\pi_{\ell}^{r}$, hence the projection $\mathbb{E}_{1} \rightarrow M$ restricted to the manifold $W_{\ell}^{r}$ is the standard covering of $M$ associated to the subgroup $\pi_{\ell}^{r}$.

Each fiber of $\mathbb{E}_{1} \rightarrow M$ over $x \in M$ is naturally isometric to the unit sphere $\mathbb{S}^{m+n-1} \subset \mathbb{V}$, and is given the induced Riemannian metric with geodesic distance function $d_{x}^{v}$, so has circumference $2 \pi$. Given any pair of orthogonal unit vectors $\vec{v}, \vec{u} \in \mathbb{V}$, we have $d_{x}^{v}(\vec{v}, \vec{u})=\pi / 2>1$.

The submanifolds $W_{\ell}^{s}$ and $W_{\ell}^{r}$ intersect the fiber of $\mathbb{E}_{1} \rightarrow M$ over $x$ in points corresponding to the orbits $\mathcal{O}_{\ell}^{s}$ and $\mathcal{O}_{\ell}^{r}$. Thus, for distinct points $z, w \in \mathcal{O}_{\ell}^{s} \cup \mathcal{O}_{\ell}^{r}$, the distance $d_{\mathbb{S}^{p}}(z, w)=\pi / 2>1$. By construction, for $\ell \neq \lambda$, the vector subspaces $\mathbb{V}_{\ell}^{s}, \mathbb{V}_{\ell}^{r}, \mathbb{V}_{\lambda}^{s}$ and $\mathbb{V}_{\lambda}^{r}$ are all pairwise orthogonal. Thus, if $z \in \mathcal{O}_{\ell}^{s} \cup \mathcal{O}_{\ell}^{r}$ and $w \in \mathcal{O}_{\lambda}^{s} \cup \mathcal{O}_{\lambda}^{r}$, then $d_{\mathbb{S}^{p}}(z, w)=\pi / 2$.

Define the inclusion of the sphere bundle $\mathbb{E}_{1}$ into $N_{0}$ via the composition

$$
\iota_{0}: \mathbb{E}_{1} \subset \mathbb{E} \subset \mathbb{E} \oplus \mathbb{F} \oplus \varepsilon \cong \varepsilon^{p+1} .
$$

Define $W_{\ell, 0}^{s} \subset N_{0}$ and $W_{\ell, 0}^{r} \subset N_{0}$ as the images under the map $\iota_{0}$ of (11) of $W_{\ell}^{s}$ and $W_{\ell}^{r}$ respectively, so we obtain diffeomorphisms $\iota_{\ell}^{s}: W_{\ell}^{s} \rightarrow W_{\ell, 0}^{s}$ and $\iota_{\ell}^{r}: W_{\ell}^{r} \rightarrow W_{\ell, 0}^{r}$.

Let $\vec{n}_{0}: M \rightarrow N_{0}$ be the north-pole section defined by $\vec{n}_{0}(x)=\{x\} \times$ $(0, \ldots, 0,1)$. Similarly, let $\vec{s}_{0}: M \rightarrow N_{0}$ be the south-pole section defined by $\vec{s}_{0}(x)=\{x\} \times(0, \ldots, 0,-1)$. The manifold $N_{0}$ with the north-pole section deleted is

$$
N_{0}-\vec{n}_{0}(M)=M \times\left\{\mathbb{S}^{p}-(0, \ldots, 0,1)\right\} \cong M \times \mathbb{R}^{p},
$$

where the last isomorphism uses stereographic projection from the south pole in each fiber. For each $z \in N_{0}-\vec{n}_{0}(M)$ the identification (21) induces a framing of the fiberwise tangent space $T_{z}^{v} N_{0}$ of $N_{0}$ at $z$.

Each fiber $\{x\} \times \mathbb{S}^{p}$ of $N_{0}=M \times \mathbb{S}^{p}$ over $x \in M$ has the standard Riemannian metric with geodesic distance function denoted by $d_{\mathbb{S}^{p}}$, and with circumference $2 \pi$. The inclusion $\iota_{0}: \mathbb{E}_{1} \rightarrow N_{0}$ is a fiberwise isometric embedding, and the image of $\iota_{0}$ is fiberwise orthogonal to the section $\vec{n}$. Hence, for each $x \in M$, the submanifolds $W_{\ell}^{s}$ and $W_{\ell}^{r}$ intersect the fiber of $N_{0} \rightarrow M$ over $x$ in points which are fiberwise orthogonal to $\vec{n}(x)$. Let 
$W_{\ell, x}^{s}=W_{\ell}^{s} \cap\left(\{x\} \times \mathbb{S}^{p}\right)$ and $W_{\ell, x}^{r}=W_{\ell}^{r} \cap\left(\{x\} \times \mathbb{S}^{p}\right)$. Then for each point $z \in W_{\ell, x}^{s}$ or $W_{\ell, x}^{r}$ the fiberwise distance to the north pole $\vec{n}_{0}(x)$ is $\pi / 2$.

Let $0<\epsilon<\pi / 4$, then each $x \in M$ and $1 \leq \ell \leq k$, define the fiberwise disk neighborhoods $\mathbb{D}^{p}\left(W_{\ell, x}^{s}, \epsilon\right)$ and $\mathbb{D}^{p}\left(W_{\ell, x}^{r}, \epsilon\right)$ of $W_{\ell, x}^{s}$ and $W_{\ell, x}^{r}$ as in (13). Their boundaries $\mathbb{S}^{p-1}\left(W_{\ell, x}^{s}, \epsilon\right)$ and $\mathbb{S}^{p-1}\left(W_{\ell, x}^{r}, \epsilon\right)$ are defined as in (14).

Fix $\epsilon=1 / 10$, then define the open tubular neighborhoods $\mathbb{D}^{p}\left(W_{\ell}^{s}\right)$ and $\mathbb{D}^{p}\left(W_{\ell}^{r}\right)$ of $W_{\ell}^{s}$ and $W_{\ell}^{r}$ in $N_{0}$ as in (15). Their boundaries $T_{\ell}^{s} \subset N_{0}$ and $T_{\ell}^{r} \subset N_{0}$ are defined as in (16).

\subsection{Construction of the foliation}

Set

$$
N_{1}=N_{0}-\left(\mathbb{D}^{p}\left(W_{1}^{s}\right) \cup \mathbb{D}^{p}\left(W_{1}^{r}\right) \cup \cdots \cup \mathbb{D}^{p}\left(W_{k}^{s}\right) \cup \mathbb{D}^{p}\left(W_{k}^{r}\right)\right) .
$$

The boundary of $N_{1}$ has $2 k$ connected components,

$$
\partial N_{1}=\partial_{1}^{s} N_{1} \cup \partial_{1}^{r} N_{1} \cup \cdots \cup \partial_{k}^{s} N_{1} \cup \partial_{k}^{r} N_{1},
$$

where $\partial_{\ell}^{s} N_{1}=T_{\ell}^{s}$ and $\partial_{\ell}^{r} N_{1}=T_{\ell}^{r}$. The manifold $N_{1}$ fibers over $M$, defining a foliation $\mathcal{F}_{0}$. The fiber of $N_{1}$ over $x \in M$ is the set

$$
\begin{aligned}
& \mathbf{P}_{\ell, x}^{p}=N_{1} \cap\left(\{x\} \times \mathbb{S}^{p}\right)=\left(\{x\} \times \mathbb{S}^{p}\right) \\
& -\left(\mathbb{D}^{p}\left(W_{\ell, x}^{s}, 1 / 10\right) \cup \mathbb{D}^{p}\left(W_{\ell, x}^{r}, \epsilon\right) \cup \cdots \cup \mathbb{D}^{p}\left(W_{\ell, x}^{s}, 1 / 10\right) \cup \mathbb{D}^{p}\left(W_{\ell, x}^{r}, \epsilon\right)\right) .
\end{aligned}
$$

The typical leaf of $\mathcal{F}_{0}$ is diffeomorphic to the sphere $\mathbb{S}^{p}$ with $m+n=m_{1}+$ $\cdots+m_{k}+n_{1}+\cdots+m_{k}$ disks removed, and the dimension is $p=m+n+\xi$.

Each submanifold $T_{\ell}^{s}$ is disjoint from the north pole section $\vec{n}_{0}$ so the fibers of the map $T_{\ell}^{s} \rightarrow M$ are trivialized by the map (21). The similar statement holds for $T_{\ell}^{r}$, so we obtain fiberwise identifications

$$
\begin{aligned}
& \varphi_{\ell}^{s}: T_{\ell}^{s} \cong W_{\ell, 0}^{s} \times \mathbb{S}^{p-1}, \\
& \varphi_{\ell}^{r}: T_{\ell}^{r} \cong W_{\ell, 0}^{r} \times \mathbb{S}^{p-1} .
\end{aligned}
$$

For each $1 \leq \ell \leq k$, we are given the diffeomorphism $h_{\ell}: P_{\pi_{\ell}^{s}} \rightarrow P_{\pi_{\ell}^{r}}$ where $P_{\pi_{\ell}^{s}}$ is standard, so canonically identified with $W_{\ell}^{s}$ and hence with $W_{\ell, 0}^{s}$, while $P_{\pi_{\ell}^{r}}$ is identified with $W_{\ell, 0}^{r}$. Thus, $h_{\ell}$ induces a diffeomorphism

$$
H_{\ell}=\left(\varphi_{\ell}^{r}\right)^{-1} \circ\left(h_{\ell} \times \mathrm{Id}\right) \circ \varphi_{\ell}^{s}: T_{\ell}^{s} \rightarrow T_{\ell}^{r},
$$

which maps fibers to fibers. That is, $H_{\ell}$ preserves the foliation on the boundary components $\partial_{\ell}^{s} N_{1}$ and $\partial_{\ell}^{r} N_{1}$ of $N_{1}$ induced by $\mathcal{F}_{0}$.

Define $N=N_{1} / \sim$, where we identify $H_{\ell}: T_{\ell}^{s} \sim T_{\ell}^{r}$ for each component. Let $\mathcal{F}_{\mathcal{C}}$ be the foliation whose leaves are obtained from the those of $\mathcal{F}_{0}$ by 
the identification maps $H_{\ell}$. This completes the construction of the "Hirsch foliation" realizing the family of étale correspondences $\mathcal{C}$.

The manifold $M$ has a natural embedding $M_{0}=\vec{s}(M)$ into $N$ as the image of the south pole section of $M \times \mathbb{S}^{p}$, and the holonomy pseudogroup of $\mathcal{F}_{\mathcal{C}}$ induced on $M_{0}$ is equivalent to the pseudogroup $\Gamma_{\mathcal{C}}$ as defined in Section 3.6. Hence, the dynamics of $\mathcal{F}_{\mathcal{C}}$ induced on the section $M_{0}$ is equivalent to the dynamics of $\Gamma_{\mathcal{C}}$ acting on $M$.

Note that the leaves of $\mathcal{F}_{\mathcal{C}}$ have even more complicated topology as immersed submanifolds of $N$ than in the case of a single étale correspondence. Again, it would be quite interesting to understand the foliated coarse cohomology of these Hirsch foliations, and whether the topology of the embeddings of the leaves into $N$ are part of the data required to calculate the cohomology groups.

\section{Examples}

In this section, we will give three examples of generalized Hirsch foliations.

Example 6.1 Markov minimal sets in codimension one

A Markov system is a special class of 1-dimensional dynamical system, which has fundamental importance in the study of codimension one foliations. The most general definition has been given by Takashi Inaba and Shigenori Matsumoto. We recall their definition from Section 5 of [43].

Definition 6.2 Let $T$ be a compact 1-dimensional manifold, and $r \geq 0$. A $C^{r}$ Markov Minimal Set $\mathbf{K} \subset T$ is a closed nowhere dense subset such that

1. there are closed intervals $I_{i} \subset T$ for $1 \leq i \leq k$,

2. $\operatorname{Int}\left(I_{i}\right) \cap \operatorname{Int}\left(I_{j}\right)=\emptyset$ for $i \neq j$,

3. $\mathbf{K} \subset I_{1} \cup \cdots \cup I_{k}$,

4. $\mathbf{K} \cap \operatorname{Int}\left(I_{i}\right) \neq \emptyset$ for all $1 \leq i \leq k$,

5. there is an open interval $U_{i}$ with $I_{i} \subset U_{i}$ and a $C^{r}$-diffeomorphism onto its image $h_{i}: U_{i} \rightarrow T$,

6. if $h_{i}\left(I_{i}\right) \cap \operatorname{Int}\left(I_{j}\right) \neq \emptyset$, then $I_{j} \subset h_{i}\left(I_{i}\right)$,

7. $\mathbf{K}$ is a minimal set for the dynamical system given by the pseudogroup $\Gamma$ modeled on $T$ generated by the maps $\left\{h_{1}, \ldots, h_{k}\right\}$.

Note that the 1-manifold $T$ need not be connected, though typically one takes either $T=[0,1] \subset \mathbb{R}$ or $T=\mathbb{S}^{1}$. The definition of a Markov 
Minimal Set in $[11,12,58]$ replaces condition (6.2.2) above with the stronger hypothesis

$$
2^{\prime} I_{i} \cap I_{j}=\emptyset \text { for } i \neq j \text {. }
$$

Section 6 of [43] gives a construction of exceptional minimal sets which only satisfy this more general definition, in that the natural Markov partition cannot be chosen to consist of disjoint closed intervals. The papers [13, 42] give constructions of foliations realizing a Markov Minimal Set satisfying the stronger condition (6.2.2').

We show here how to realize a special case of a $C^{r}$-Markov system (one for which $I_{j} \subset h_{i}\left(I_{i}\right)$ for all $\left.i, j\right)$ using the Hirsch construction of Section 2.2. We assume there is given the following data:

- $I_{0}=\left[a_{0}, b_{0}\right], I_{1}=\left[a_{1}, b_{1}\right], \ldots, I_{k}=\left[a_{k}, b_{k}\right], I_{i} \subset I_{0}$ for all $1 \leq i \leq k$,

- $I_{i} \cap I_{j}=\emptyset$ for $i \neq j$ and $i, j \neq 0$,

- $C^{r}$ expansive maps $\psi_{i}: I_{i} \rightarrow I_{0}, 1 \leq i \leq k$.

For $r \geq 1$ we can require that the maps $\psi_{i}$ satisfy $\psi_{i}^{\prime}(x)>1$ for $x \in I_{i}$ in which case it is called a hyperbolic Markov system. The pseudogroup generated by the maps $\left\{\psi_{1}, \ldots \psi_{k}\right\}$ has an exceptional minimal set $\mathbf{K} \subset I_{0}$ which is characterized by the condition

$$
\mathbf{K}=\psi_{1}^{-1}(\mathbf{K}) \cup \cdots \cup \psi_{k}^{-1}(\mathbf{K}) .
$$

We first normalize the given data. The endpoints of the intervals are labeled in increasing order:

$$
a_{0}<a_{1}<b_{1}<a_{2}<\cdots<b_{k-1}<a_{k}<b_{k}<b_{0} .
$$

We are interested in the realization of the minimal set $\mathbf{K} \subset I_{0}$, so we can assume both $a_{0} \in \mathbf{K}$ and $b_{0} \in \mathbf{K}$, as otherwise we simply restrict the domain $I_{0}$ so that $a_{0}$ is the least fixed-point of $\psi_{1}: I_{1} \rightarrow I_{0}$, and $b_{0}$ is the greatest fixed-point of $\psi_{k}: I_{k} \rightarrow I_{0}$. The holonomy pseudogroup is only defined up to $C^{r}$-diffeomorphism, so without loss of generality we can assume that $a_{0}=0$ and $0<b_{0}<1$, so $I_{0} \subset[0,1)$.

Following the construction in Section 2.2, we need to choose an immersion $H: \mathbb{S}^{1} \rightarrow \mathbb{S}^{1}$ of degree $k$, which is equivalent to the choice of a diffeomorphism $h: \mathbb{R} \rightarrow \mathbb{R}$ such that $h(x+1)=h(x)+k$. Then $H=h$ $\bmod (1)$. Figure 5 below illustrates the definition of $h:[0,1] \rightarrow[0,3]$ for $k=3$. 


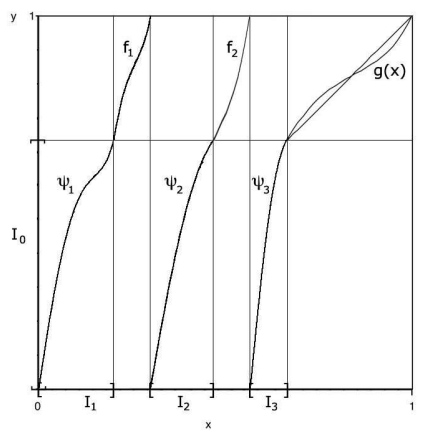

Figure 5. 3-1 map realizing Markov system

The formal definition is as follows:

$$
h(x)= \begin{cases}\psi_{i}(x) & \text { if } a_{i} \leq x \leq b_{i}, 1 \leq i \leq k, \\ f_{i}(x)+(i-1) & \text { if } b_{i} \leq x \leq a_{i+1}, 1 \leq i<k, \\ g(x)+(k-1) & \text { if } b_{k} \leq x \leq 1\end{cases}
$$

where $f_{i}:\left[b_{i}, a_{i+1}\right] \rightarrow\left[b_{0}, 1\right]$ is a $C^{r}$-diffeomorphism onto, chosen so that $h$ is $C^{r}$ at the points $b_{i}$ and $a_{i+1}$. The map $g:\left[b_{0}, 1\right] \rightarrow\left[b_{0}, 1\right]$ is as pictured, a $C^{r}$-contraction on the open interval $\mathcal{J}=\left(b_{0}, 1\right)$ with a unique attracting fixed-point at $z_{0}=\left(b_{0}+1\right) / 2$. The map $g$ satisfies $g\left(b_{0}\right)=b_{0}$ and $g(1)=1$, and is chosen so that the resulting map $h$ is $C^{r}$ at the endpoints $b_{0}$ and 1.

Define the open set $U \subset \mathbb{S}^{1}$ to be the union of the orbits of the open interval $\mathcal{J}$, and $\mathbf{K}=\mathbb{S}^{1}-U$. Then $\mathbf{K} \subset I_{1} \cup \cdots \cup I_{k}$.

The proofs that $\mathbf{K}$ is non-empty, nowhere dense, and that the orbit of every point in $\mathbf{K}$ is dense in $\mathbf{K}$, are all exactly the same as in Section 2.6.

Example 6.3 Sierpinski carpet minimal sets in codimension two.

This example constructs a smooth $4-1$ covering map $h: \mathbb{T}^{2} \rightarrow \mathbb{T}^{2}$ with a unique exceptional minimal set that is homeomorphic to the "Sierpinski torus", which is obtained from the traditional Sierpinski carpet pictured below by identifying opposites edges.

Let $h_{0}: \mathbb{T}^{2} \rightarrow \mathbb{T}^{2}$ be the "2-times" map, defined as the quotient of the covering map $f_{0}: \mathbb{R}^{2} \rightarrow \mathbb{R}^{2}$, with $f_{0}(\vec{x})=2 \vec{x}$. The dynamics of the map $h_{0}$ is well-known - it is minimal with positive entropy. Both statements are consequences of the observation that $h_{0}$ admits a "Markov partition". This idea plays an important role in our example, so we recall the construction. 


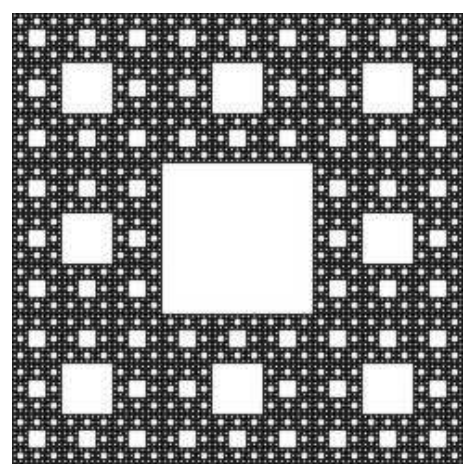

Figure 6. Classic Sierpinski Carpet

For each pair of integers $m, n \in \mathbb{Z}$, the unit square

$$
S_{m, n}=\{(x, y) \mid m \leq x \leq m+1, n \leq y \leq n+1\} \subset \mathbb{R}^{2}
$$

is a fundamental domain for $\mathbb{T}^{2}=\mathbb{R}^{2} / \mathbb{Z}^{2}$. The $h_{0}$ has four "inverse maps",

$$
\begin{aligned}
& g_{1}=h_{00}^{-1}: S_{00} \rightarrow P_{1}=\{(x, y) \mid 0 \leq x \leq 1 / 2,0 \leq y \leq 1 / 2\}, \\
& g_{2}=h_{10}^{-1}: S_{10} \rightarrow P_{2}=\{(x, y) \mid 1 / 2 \leq x \leq 1,0 \leq y \leq 1 / 2\}, \\
& g_{3}=h_{01}^{-1}: S_{01} \rightarrow P_{3}=\{(x, y) \mid 0 \leq x \leq 1 / 2,1 / 2 \leq y \leq 1\}, \\
& g_{4}=h_{11}^{-1}: S_{11} \rightarrow P_{4}=\{(x, y) \mid 1 / 2 \leq x \leq 1,1 / 2 \leq y \leq 1\},
\end{aligned}
$$

where the interiors of the four partitions $P_{i}$ of $S_{00}$ are disjoint. Given a word $I=\left(i_{1}, \ldots, i_{n}\right)$ of length $\|I\|=n$, where each $i_{j} \in\{1,2,3,4\}$, form the composition $g_{I}=g_{i_{n}} \circ \cdots g_{i_{1}}: S_{00} \rightarrow P_{I}$ where $P_{I}$ is a square of side length $2^{-n}$. Given any point $z \in S_{00}$ the images $\left\{g_{I}(z) \mid\|I\|=n\right\}$ form a net in $S_{00}$ whose distance between points is $\sqrt{2} / 2^{n}$. This implies the orbit of $z$ under the dynamics generated by $h_{0}$ is dense in $\mathbb{T}^{2}$, and that the topological entropy of the system is $\ln 4$.

The map $h: \mathbb{T}^{2} \rightarrow \mathbb{T}^{2}$ is obtained by introducing a sink for the map $h_{0}$, in a manner exactly analogous to the construction of the original Hirsch foliation from the affine $2-1$ Hirsch foliation. In fact, the map $h$ agrees with the map $h_{0}$ on three of the four fundamental partitions: $h_{0}\left|P_{i}=h_{0}\right| P_{i}$ for $i=1,2,3$.

We describe the map $h: P_{4} \rightarrow S_{11}$ on the fourth partition. Let $U_{0} \subset P_{4}$ be the open set defined by

$$
U_{0}=\{(x, y) \mid 5 / 8<x<7 / 8,5 / 8<y<7 / 8\} .
$$


Let $\phi: \overline{U_{0}} \rightarrow \overline{U_{0}}$ be a smooth diffeomoprhism which is the identity on the boundary $\partial \overline{U_{0}}=\overline{U_{0}}-U_{0}$, and on the interior $U_{0}$ is a contraction to a fixed-point $x_{0}=(3 / 4,3 / 4)$.

Let $\psi: P_{4}-U_{0} \rightarrow S_{00}-U_{0}$ be a smooth diffeomoprhism which agrees with the expanding map $(x, y) \mapsto(2 x-1,2 y-1)$ on the outside boundary $\partial P_{4}$ of $P_{4}-U_{0}$, agrees with the identity map on the inside boundary $\partial \overline{U_{0}}$ of $P_{4}-U_{0}$, and is expanding on the interior of $P_{4}-U_{0}$. Define $f: P_{4} \rightarrow S_{11}$ by

$$
h(x, y)= \begin{cases}\phi(x, y)+(1,1) & \text { if }(x, y) \in U_{0}, \\ \psi(x, y)+(1,1) & \text { if }(x, y) \in P_{4}-U_{0} .\end{cases}
$$

Then $f: S_{00} \rightarrow S_{00} \cup S_{10} \cup S_{01} \cup S_{11}$ is a smooth diffeomorphism onto, and satisfies the Markov partition conditions (22-25) by construction. Let $h: \mathbb{T}^{2} \rightarrow \mathbb{T}^{2}$ be the $4-1$ map induced by $f$.

Let $U$ be the union of all orbits of points in $U_{0}$ for the dynamical system generated by $h$. Let $\mathbf{K}=\mathbb{T}^{2}-U$, then $\mathbf{K}$ is a closed invariant subset for the dynamical system of $h$, and it is not hard to see that $\mathbf{K}$ is minimal, using the Markov structure of $h$.

This example admits many generalizations, which are discussed in [5] along with many of their properties. It is also interesting to compare this construction with the methods of [7] where the authors construct homeomorphisms with a Sierpinski 2-torus as a unique minimal set. These examples provide a general solution of Problem 4 of [7].

Example 6.4 Affine foliation of codimension $q$ with local holonomy $\mathbf{S L}(q, \mathbb{Z})$.

Let $\Gamma \subset \mathbf{S L}(q, \mathbb{Z})$ be a finitely generated subgroup; or rather, for matrices $\left\{A_{1}, \ldots, A_{k}\right\} \subset \mathbf{S L}(q, \mathbb{Z})$ let $\Gamma$ denote the group they generate. For each index $1 \leq \ell \leq k$, let $\lambda_{\ell} \in \mathbb{N}$ be a positive integer, and let $\Lambda_{\ell}=\lambda_{\ell} \cdot I d$ be the diagonal matrix with all diagonal entries $\lambda_{\ell}$. Let $B_{\ell}=\Lambda_{\ell} \cdot A_{\ell}$ be the integer matrix with inverse $B_{\ell}^{-1} \in \mathbf{S L}(q, \mathbb{Q})$.

An integer matrix $C$ determines an affine map $\widetilde{C}: \mathbb{T}^{q} \rightarrow \mathbb{T}^{q}$ which is the quotient of the multiplication map $C: \mathbb{R}^{q} \rightarrow \mathbb{R}^{q}$, where $\mathbb{T}^{q}=\mathbb{R}^{q} / \mathbb{Z}^{q}$. For each $\ell$ we have the commutative diagram

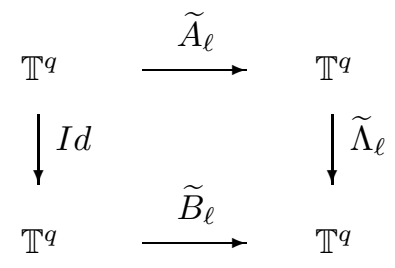


which defines an étale correspondence $\left(s_{\ell}, r_{\ell}, h_{\ell}\right)=\left(I d, \widetilde{\Lambda}_{\ell}, \widetilde{A}_{\ell}\right)$ where $P_{\ell}=$ $Q_{\ell}=M=\mathbb{T}^{q}$ and the covering indices are $m_{\ell}=1, n_{\ell}=\lambda_{\ell}^{q}$. This yields a system of étale correspondences as in Section 5 ,

$$
\begin{aligned}
& \mathcal{C}= \\
& \left\{\left(s_{\ell}=I d: \mathbb{T}^{q} \rightarrow \mathbb{T}^{q}, r_{\ell}=\widetilde{\Lambda}_{\ell}: \mathbb{T}^{q} \rightarrow \mathbb{T}^{q}, h_{\ell}=\widetilde{A}_{\ell}: \mathbb{T}^{q} \rightarrow \mathbb{T}^{q}\right) \mid 1 \leq \ell \leq k\right\} .
\end{aligned}
$$

The construction in Section 5 then yields a foliation $\mathcal{F}_{\mathcal{C}}$ of codimension $q$ with transversal $\mathbb{T}^{q}$ whose global holonomy induced on the section $M_{0}=\mathbb{T}^{q}$ is equivalent to the pseudogroup $\Gamma_{\mathcal{C}}$ generated by the maps $\left\{\widetilde{B}_{\ell}: \mathbb{T}^{q} \rightarrow \mathbb{T}^{q} \mid\right.$ $1 \leq \ell \leq k\}$.

A special case of the above construction occurs for $\Gamma=\mathbf{S L}(q, \mathbb{Z})$ and $\left\{A_{1}, \ldots, A_{k}\right\}$ is a set of generators. Note that for any pair $1 \leq i, j \leq k$ we have that

$$
\left[B_{i}, B_{j}\right]=B_{i} B_{j} B_{i}^{-1} B_{j}^{-1}=A_{i} A_{j} A_{i}^{-1} A_{j}^{-1}=\left[A_{i}, A_{j}\right]
$$

as the factors $\Lambda_{i}$ and $\Lambda_{j}$ are multiples of the identity. Thus, the subgroup $\widehat{\Gamma}=\left\langle B_{1}, \ldots, B_{k}\right\rangle \subset \mathbf{S L}(q, \mathbb{Q})$ generated by the matrices $\left\{B_{\ell}\right\}$ contains a subgroup isomorphic to the commutator subgroup $[\Gamma, \Gamma] \subset \mathbf{S L}(q, \mathbb{Z})$. (We thank Alex Furman for this observation.) It is elementary that $[\Gamma, \Gamma]$ is a normal subgroup of finite index in $\mathbf{S L}(q, \mathbb{Z})$.

While the commutator $\left[\widetilde{B}_{i}, \widetilde{B}_{j}\right]$ of maps is not well-defined as diffeomorphisms of $\mathbb{T}^{q}$, it is well-defined as local elements of the holonomy groupoid $\Gamma_{\mathcal{C}}$. Thus, the holonomy groupoid $\Gamma_{\mathcal{C}}$ contains a subgroupoid equivalent to that generated by the action of $[\Gamma, \Gamma]$ on $\mathbb{T}^{q}$. So, in a sense, $\Gamma_{\mathcal{C}}$ is a virtual congruence subgroup of $\mathbf{S L}(q, \mathbb{Z})$ (in the sense of George Mackey [46, 47, 51] that holonomy pseudogroups represent virtual subgroups.)

Conjecture 6.5 For $q \geq 3$, and $\Gamma \subset \mathbf{S L}(q, \mathbb{Z})$ finite index, then for any choice of generators $\left\{A_{1}, \ldots, A_{k}\right\} \subset \Gamma$ and positive integers $\left\{\lambda_{1}, \ldots, \lambda_{k}\right\}$, the foliation $\mathcal{F}_{\mathcal{C}}$ as constructed above is $C^{1}$-structurally stable.

Note that for all $\lambda_{\ell}=1$, the foliation $\mathcal{F}_{\mathcal{C}}$ is the suspension of the group action of $\Gamma$ on $\mathbb{T}^{q}$ so this case follows by the general theory of $C^{1}$-rigidity of actions of higher rank lattices (see [21] for the latest results in this area.)

The methods of $[37,38,40]$ suffice to prove the foliations $\mathcal{F}_{\mathcal{C}}$ are stable under $C^{1}$ deformations; details will appear in [41].

\section{Some Questions}

It seems clear that the examples of smooth foliations constructed with the generalized Hirsch method realize a wide range of dynamical behavior on 
$M$, as expanding maps provide one of the main sources of hyperbolic and chaotic behavior in dynamical systems, and these are just some part of the possible maps in a system of étale correspondences.

Question 7.1 Given a compact manifold $M$ without boundary, and a connected continua $\mathbf{K} \subset M$, is there a system of étale correspondences $\mathcal{C}$ on $M$ for which $\mathbf{K}$ is a minimal set for the associated pseudogroup $\Gamma_{\mathcal{C}}$ ?

The only known "obstruction" is that a minimal set must be "locally homogeneous", in that every orbit is dense so any locally-defined property of $\mathbf{K}$ must occur at a dense set of points in $\mathbf{K}$.

There is a variant on this question which seems worth emphasizing. Suppose that $M$ is a closed 3-manifold which admits a proper self-covering $h: M \rightarrow M$. Then for every pair of diffeomorphisms $f, g: M \rightarrow M$ the composition $g \circ h \circ f: M \rightarrow M$ is again a proper self-covering. Every minimal set $\mathbf{K} \subset M$ for the dynamics of the map $g \circ h \circ f$ will have positive entropy (see [5]) so the minimal set $\mathbf{K}$ has non-trivial dynamical complexity.

Question 7.2 Can one characterize the geometry of the minimal set $\mathbf{K}$ for $g \circ h \circ f$ in terms of the topology of the 3-manifold $M$ ?

The point is that the topology of a 3-manifold $M$ which admits a selfmap should be closely related to the dynamics of a self-map of $M$ of higher degree. For example, if $M$ is a Seifert manifold, then must the minimal set $\mathbf{K}$ have a fibration into continua of dimension one?

We say a foliated manifold $(M, \mathcal{F})$ is co-Hopfian if a foliated covering map $h: M \rightarrow M$ is necessarily a diffeomorphism.

Question 7.3 Which foliations are co-Hopfian?

There are two obvious ways to construct a foliation which is not coHopfian: a foliated covering map $h: M \rightarrow M$ can be chosen to be "expanding" along leaf directions, or along transverse directions. Is this always the case? Does a non-co-Hopf map have degree which factors into tangential and transverse degrees?

Haefliger has posed the problem of determining which compactly generated pseudogroups can be realized as the pseudogroup of a foliation on a closed manifold [31].

Question 7.4 Given a compact manifold $M$ without boundary, is there a general description of the pseudogroups modeled on $M$ which can be realized up to pseudogroup equivalence by a system of étale correspondences?

One does not expect a ready answer to such a question, but it is completely unknown just how large a class of pseudogroups are represented by those equivalent to one of the type $\Gamma_{\mathcal{C}}$ for some system of étale correspondences $\mathcal{C}$. Of course, if $M$ is simply connected, this is just asking 
which pseudogroups on $M$ can be realized by a finitely-generated group of diffeomorphisms, to which there is also no known answer.

\section{Acknowledgments}

Andrzej Biś was supported by a Marie Curie International Fellowship within the $6^{\text {th }}$ European Community Framework Programm and Steven Hurder was supported in part by NSF grant DMS-0406254.

\section{References}

1. D. Andrica and L. Funar, On smooth maps with finitely many critical points, J. London Math. Soc. (2), 69 (2004), 783-800.

2. R. Baer, Groups without proper isomorphic quotient groups, Bull. Amer. Math. Soc., 50 (1944), 267-278.

3. I. Belegradek, On co-Hopfian nilpotent groups, Bull. London Math. Soc., 35 (2003), 805-811.

4. A. Bís and S. Hurder, Lattice actions on Sierpinski manifolds, preprint, 2006.

5. A. Bís and S. Hurder, Markov minimal sets of foliations, preprint, 2006.

6. A. Biś, H. Nakayama and P. Walczak, Locally connected exceptional minimal sets of surface homeomorphisms, Ann. Inst. Fourier (Grenoble), 54 (2003), 711-731.

7. A. Biś, H. Nakayama and P. Walczak, Modeling minimal foliated spaces with positive entropy, preprint, 2003.

8. S.V. Buyalo and P.V. Svetlov, Topological and geometric properties of graph manifolds, Algebra i Analiz., 16 (2004), 3-68.

9. C. Camacho and A. Lins Neto, Geometric Theory of Foliations, Translated from the Portuguese by Sue E. Goodman, Progress in Mathematics, Birkhäuser Boston, MA, 1985.

10. A. Candel and L. Conlon, Foliations I, Amer. Math. Soc., Providence, RI, 2000.

11. J. Cantwell and L. Conlon, Foliations and subshifts, Tohoku Math. J., 40 (1988), 165-187.

12. J. Cantwell and L. Conlon, Leaves of Markov local minimal sets in foliations of codimension one, Publications Matematiques, Universitat Autòmata de Barcelona, 33 (1989), 461-484.

13. J. Cantwell and L. Conlon, Endsets of exceptional leaves; a theorem of G. Duminy, in Foliations: Geometry and Dynamics (Warsaw, 2000), 
World Scientific Publishing Co. Inc., River Edge, NJ, 2002, 225-261.

14. K. Dekimpe and K.-B. Lee, Expanding maps on infra-nilmanifolds of homogeneous type, Trans. Amer. Math. Soc., 355 (2003), 1067-1077.

15. K. Dekimpe and K.-B. Lee, Expanding maps, Anosov diffeomorphisms and affine structures on infra-nilmanifolds, Topology Appl., 130 (2003), 259-269.

16. A.L. Delgado and M. Timm, Spaces whose finite sheeted covers are homeomorphic to a fixed space, Topology Appl., 129 (2003), 1-10.

17. T. Delzant and L. Potyagailo, Endomorphisms of Kleinian groups, Geom. Funct. Anal., 13 (2003), 396-436.

18. B. Deroin, Almost-holomorphic and totally real laminations in complex surfaces, preprint, Max-Planck-Institut für Mathematik, 2005.

19. B. Farb and J. Franks, Group actions on one-manifolds. II. Extensions of Hölder's theorem, Trans. Amer. Math. Soc., 355 (2003), 43854396.

20. B. Farb and J. Franks, Groups of homeomorphisms of one-manifolds. III. Nilpotent subgroups, Ergodic Theory Dynam. Systems, 23 (2003), $1467-1484$.

21. D. Fisher and G. Margulis, Local rigidity of affine actions of higher rank groups and lattices, preprint, 2005.

22. É. Ghys, R. Langevin, and P. Walczak, Entropie géométrique des feuilletages, Acta Math., 160 (1988), 105-142.

23. C. Godbillon, Feuilletages: Etudes géométriques I, II, Publ. IRMA Strasbourg (1985-86); Progress in Math., 98, Birkhäuser, Boston, Mass., 1991.

24. F. González-Acuña, R. Litherland and W. Whitten, Co-Hopficity of Seifert-bundle groups, Trans. Amer. Math. Soc., 341 (1994), 143155.

25. F. González-Acuña and W. Whitten, Imbeddings of three-manifold groups, Mem. Amer. Math. Soc., 99, no. 474, 1992.

26. F. González-Acuña and W. Whitten, Co-Hopficity of 3-manifold groups, Topology Appl., 56 (1994), 87-97.

27. M. Gromov, Groups of polynomial growth and expanding maps, Inst. Hautes Études Sci. Publ. Math., 53 (1981), 53-73.

28. M. Gromov, Volume and bounded cohomology, Inst. Hautes Études Sci. Publ. Math., 56 (1982), 5-99.

29. A. Haefliger, Homotopy and integrability in Manifolds-Amsterdam 1970 (Proc. Nuffic Summer School), Lecture Notes in Math., 197, Springer-Verlag, Berlin, 1971, 133-163. 
30. A. Haefliger, Groupoïdes d'holonomie et classifiants in Transversal structure of foliations (Toulouse, 1982), Asterisque, 177-178 70-94, Société Mathématique de France, 1984.

31. A. Haefliger, Foliations and compactly generated pseudogroups, in Foliations: Geometry and Dynamics (Warsaw, 2000), World Scientific Publishing Co. Inc., River Edge, N.J., 2002, 275-295.

32. G. Hector, Architecture of $C^{2}$-foliations, Astérisque, 107-108 243258, Société Mathématique de France, 1983.

33. G. Hector and U. Hirsch, Introduction to the Theory of Foliations. Part A \& B, Vieweg and Sohn, Braunschweig/Wiesbaden, 1986.

34. J. Heitsch and S. Hurder, Coarse cohomology for families, Illinois J. Math., 45(2) (2001), 323-360.

35. M. Hirsch, Expanding maps and transformation groups, in Global Analysis (Proc. Sympos. Pure Math., Vol. XIV, Berkeley, Calif., 1968), Amer. Math. Soc., Providence, RI, 1970, 125-133.

36. M. Hirsch, A stable analytic foliation with only exceptional minimal sets. in Dynamical Systems, Warwick, 1974, Lect. Notes in Math., 468, Springer-Verlag, 1975, 9-10.

37. S. Hurder, Rigidity for Anosov actions of higher rank lattices, Ann. of Math. (2), 135 (1992), 361-410.

38. S. Hurder, Topological rigidity of strong stable foliations for Cartan actions, Ergodic Theory Dynam. Systems, 14 (1994), 151-167.

39. S. Hurder, Coarse geometry of foliations, in Geometric Study of Foliations, Tokyo 1993 (eds. Mizutani et al.), World Scientific Publishing Co. Inc., River Edge, N.J., 1994, 35-96.

40. S. Hurder, Infinitesimal rigidity for hyperbolic actions, J. Differential Geom., 41 (1995), 515-527.

41. S. Hurder, Structural stability for foliations of higher rank, in preparation, 2005.

42. T. Inaba, Examples of exceptional minimal sets, in A Fête of Topology, Academic Press, Boston, MA, 1988, 95-100.

43. T. Inaba and S. Matsumoto, Resilient leaves in transversely projective foliations, Journal of Faculty of Science, University of Tokyo, 37 (1990), 89-101.

44. I. Kapovich and D. Wise, On the failure of the co-Hopf property for subgroups of word-hyperbolic groups, Israel J. Math., 122 (2001), 125 147.

45. H. Lee and K.-B. Lee, Expanding maps on 2-step infra-nilmanifolds, Topology Appl., 117 (2002), 45-58. 
46. G.W. Mackey, Ergodic theory, group theory, and differential geometry, Proc. Nat. Acad. Sci. U.S.A., 50 (1963), 1184-1191.

47. G.W. Mackey, Ergodic theory and virtual groups, Math. Ann., 166 (1966), 187-207.

48. J. Milnor, Dynamics in one complex variable, Introductory lectures, Friedr. Vieweg \& Sohn, Braunschweig, 1999. preprint date: September 1991.

49. K. Ohshika and L. Potyagailo, Self-embeddings of Kleinian groups, Ann. Sci. École Norm. Sup. (4), 31 (1998), 329-343.

50. L. Potyagallo and Sh. Van, On the co-Hopficity of 3-manifold groups, Algebra i Analiz, 11 (1999), 194-220.

51. A. Ramsay, Virtual groups and group actions, Advances in Math., 6 (1971), 253-322.

52. R. Sacksteder, On the existence of exceptional leaves in foliations of codimension one, Ann. Inst. Fourier (Grenoble), 14 (1964), 221-225.

53. Z. Sela, Structure and rigidity in (Gromov) hyperbolic groups and discrete groups in rank 1 Lie groups. II, Geom. Funct. Anal., 7 (1997), 561-593.

54. V. Sergiescu and T. Tsuboi, Acyclicity of the groups of homeomorphisms of the Menger compact spaces, Amer. J. Math., 118 (1996), 1299-1312.

55. J. Shive, Conjugation Problems for Hirsch Foliations, Thesis, University of Illinois at Chicago, 2005.

56. M. Shub, Expanding maps, in Global Analysis (Proc. Sympos. Pure Math., XIV, Berkeley, Calif., 1968), Amer. Math. Soc., Providence, RI, 1970, 273-276.

57. W.P. Thurston, Three-dimensional geometry and topology. (1), Princeton Mathematical Series, 35, Princeton University Press, 1997.

58. P. Walczak, Hausdorff dimension of Markov invariant sets, Journal Math. Society of Japan, 48 (1996), 125-133.

59. S.C. Wang and Y.Q. Wu, Covering invariants and co-Hopficity of 3manifold groups, Proc. London Math. Soc. (3), 68 (1994), 203-224.

60. S.C. Wang and Q. Zhou, Embeddings of Kleinian groups with torsion, Acta Math. Sin. (Engl. Ser.), 1 (2001), 21-34.

61. S.C. Wang and F. Yu, Covering degrees are determined by graph manifolds involved, Comment. Math. Helv., 74 (1999), 238-247. 